Freiburg-THEP 05/08

PITHA 05/11

$\mathrm{UCLA} / 05 / \mathrm{TEP} / 24$

ZU-TH 15/05

hep-ph/0508254

\title{
Decays of Scalar and Pseudoscalar Higgs Bosons into Fermions: Two-loop QCD Corrections to the Higgs-Quark-Antiquark Amplitude
}

\author{
W. Bernreuther ${ }^{\mathbf{a}}$, R. Bonciani ${ }^{\mathbf{b}}$, T. Gehrmann ${ }^{\mathbf{c}}$, \\ R. Heinesch ${ }^{\mathbf{a}}$, P. Mastrolia ${ }^{\mathbf{d}}$, and E. Remiddi ${ }^{\mathrm{e}}$ \\ a Institut für Theoretische Physik, RWTH Aachen, D-52056 Aachen, Germany \\ b Fakultät für Mathematik und Physik, Albert-Ludwigs-Universität Freiburg, \\ D-79104 Freiburg, Germany \\ c Institut für Theoretische Physik, Universität Zürich, CH-8057 Zürich, Switzerland \\ d Department of Physics and Astronomy, UCLA, Los Angeles, CA 90095-1547 \\ e Dipartimento di Fisica dell'Università di Bologna, and INFN, Sezione di Bologna, I-40126 \\ Bologna, Italy
}

\begin{abstract}
As a first step in the aim of arriving at a differential description of neutral Higgs boson decays into heavy quarks, $h \rightarrow Q \bar{Q} X$, to second order in the QCD coupling $\alpha_{S}$, we have computed the $h Q \bar{Q}$ amplitude at the two-loop level in QCD for a general neutral Higgs boson which has both scalar and pseudoscalar couplings to quarks. This amplitude is given in terms of a scalar and a pseudoscalar vertex form factor, for which we present closed analytic expressions in terms of one-dimensional harmonic polylogarithms of maximum weight 4 . The results hold for arbitrary four-momentum squared, $q^{2}$, of the Higgs boson and of the heavy quark mass, $m$. Moreover we derive the approximate expressions of these form factors near threshold and in the asymptotic regime $m^{2} / q^{2} \ll 1$.
\end{abstract}

Key words: Higgs boson decays, Multi-loop calculations, QCD corrections, Heavy quark form factors.

PACS: 11.15.Bt, 12.38.Bx, 14.65.Fy, 14.65.Ha, 14.80.Bn, 14.80.Cp 


\section{Introduction}

One of the key issues of present-day particle physics is the search for Higgs bosons, that is, the search for an answer to the question whether or not the Higgs particle of the standard model (SM), or several types of Higgs bosons as predicted by SM extensions, do exist. If Higgs particles will be found at the Tevatron and/or at the LHC, then the next important goal will be the determination of its, respectively their, couplings to fermions and gauge bosons. This task may be achieved to some extent at the LHC, but it is expected that it can be carried out in detail only at a high-energy, high-luminosity linear $e^{+} e^{-}$collider. There is a vast literature on the phenomenology of (non)standard Higgs boson(s) (cf. [1,2] for a recent review), and on the interplay of hadron and lepton colliders, in particular as far as Higgs physics is concerned [3].

In this paper we are concerned with the couplings of neutral Higgs bosons to quarks (and leptons). Apart from the possibility of getting information about the top Yukawa coupling in associated production of $t \bar{t} h$, the Yukawa couplings of neutral Higgs bosons must be determined in their decays to $b \bar{b}, c \bar{c}$, and $\tau^{+} \tau^{-}$(indirect information on the Yukawa couplings can also be obtained from the branching fractions of the channels $h \rightarrow \gamma \gamma, g g$ ). If heavy non-standard Higgs bosons exist, experimental investigation of the channel $h \rightarrow t \bar{t}$ may also be feasible. The fermionic decay rates have been calculated quite precisely, both for the SM and for some of its extensions, notably the minimal supersymmetric extension (MSSM). As far as QCD corrections to the decay rates of scalar and pseudoscalar Higgs bosons into heavy quarks, $h \rightarrow Q \bar{Q}$, are concerned, the order $\alpha_{S}$ contributions were determined a long time ago [4-7], and the order $\alpha_{S}^{2}$ corrections were computed, employing quark mass expansions to various orders, by [8-15]. (The hadronic decay rate, i.e., with the gluon channel included, is known to order $\alpha_{S}^{3}$ for $m_{q}=0$ $(q \neq t)$ and $m_{h} \ll m_{t}$, see $\left.[16,17]\right)$. Results on closed quark loop insertions to the scalar $[18,19]$ and pseudoscalar [19] vertex functions at two loops are known in the literature already. The SM electroweak corrections to $h \rightarrow f \bar{f}$ were determined in [20-26] and the radiative corrections that arise within the MSSM in [27-30].

However, not only the partial decay rates but also differential distributions and, in the case of tau leptons and top quarks, also the spin polarizations and correlations of the final state fermion-antifermion pairs should be predicted as precisely as possible, since these observables contain important information about the properties of the decaying boson. For instance, neutral Higgs boson(s) may exist that are CP-violating admixtures of scalar and pseudoscalar states. In order to find out whether $h$ has quantum numbers $J^{P C}=0^{++}, 0^{-+}$, or has undefined CP parity one may employ suitable spin-spin correlations and polarization asymmetries which were proposed and computed, including radiative corrections, in [31-33]. These apply, for light and intermediate mass Higgs bosons, to $h \rightarrow \tau^{+} \tau^{-}$, while in the case of heavy Higgs bosons the channel $h \rightarrow t \bar{t}$ becomes also relevant.

We envisage a completely differential description of the (on-shell or off-shell) decays of neutral Higgs bosons to heavy quarks, $h \rightarrow Q \bar{Q} X$, to second order in the QCD coupling $\alpha_{S}$, where $Q=c, b, t$ (this includes the resonant production of Higgs bosons at a future muon collider, $\left.\mu^{+} \mu^{-} \rightarrow h \rightarrow Q \bar{Q} X\right)$. Such a differential description is necessary, as already indicated above, for the computation of many observables, especially if phase space cuts are to be taken into account. To this order in the perturbative expansion, the amplitudes for $h \rightarrow Q \bar{Q}, h \rightarrow$ $Q \bar{Q} g$, and $h \rightarrow Q \bar{Q} i j$, where $i j=g g, q \bar{q}, Q \bar{Q}$ are required within the SM - and, in addition, a suitable jet calculus, which is yet to be worked out for massive quarks at next-to-next-to- 


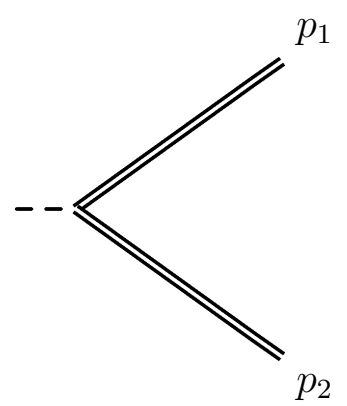

(a)

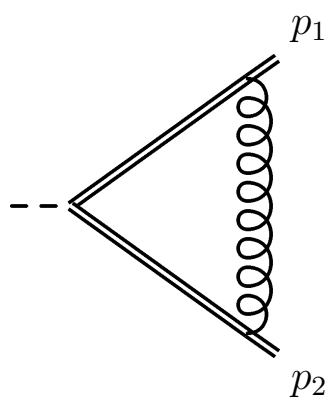

(b)

Figure 1: Tree-level and one-loop diagrams that contribute to the heavy-quark scalar and pseudoscalar form factors (3). The curly line represents a gluon, the double-line denotes the heavy quark of mass $m$. The external quarks are on their mass-shell: $p_{1}^{2}=p_{2}^{2}=m^{2}$.

leading order (NNLO) in the QCD coupling (for the case of massless quarks a NNLO subtraction method was recently developed [34], cf. also references cited therein). We consider the most general neutral Higgs boson state, i.e., a state which can couple both to scalar and pseudoscalar fermion currents. This contains scalar and pseudoscalar Higgs particles as special cases. As a first step in this project we present in this paper the $h \rightarrow Q \bar{Q}$ amplitudes to order $\alpha_{S}^{2}$ with full quark mass dependence for arbitrary squared four-momentum of the spin zero particle. Of course this yields as a special case also the second order QED corrections for the amplitudes $h \rightarrow f \bar{f}, f=$ quark or lepton.

The paper is organized as follows: In Section 2 we define the scalar and pseudoscalar vertex amplitudes for on-shell $Q, \bar{Q}$ states. These amplitudes are determined by one form factor each. Then we specify the renormalization scheme employed in this paper. In Sections 3 and 4 we give the renormalized one- and two-loop scalar and pseudoscalar form factors in the spacelike and in the timelike regions, respectively. In Section 5 and 6 we perform the threshold and asymptotic expansions, respectively, of the form factors. We conclude in Section 7.

\section{The Higgs-Quark-Antiquark Amplitude}

We consider a general neutral Higgs boson $h$ that couples both to scalar and pseudoscalar fermion currents. This includes scalar or pseudoscalar Higgs particles as special cases. The Yukawa couplings of $h$ to a massive quark $Q$ read:

$$
\mathcal{L}_{Y}=-\frac{m_{0}}{v}\left[a_{Q} \bar{Q} Q+b_{Q} \bar{Q} i \gamma_{5} Q\right] h
$$

where $Q$ and $h$ denote bare fields, $m_{0}$ is the bare mass of $Q, v=\left(\sqrt{2} G_{F}\right)^{-1 / 2}$ is the SM Higgs vacuum expectation value with $G_{F}$ being Fermi's constant, and $a_{Q}, b_{Q}$ are dimensionless "reduced" Yukawa couplings whose values depend on the parameters of the specific model under consideration. 


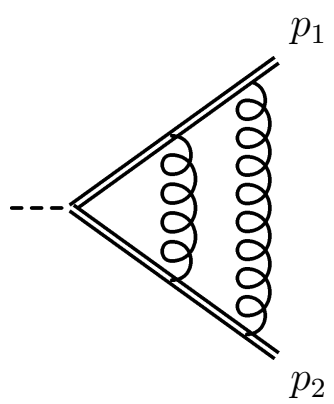

(a)

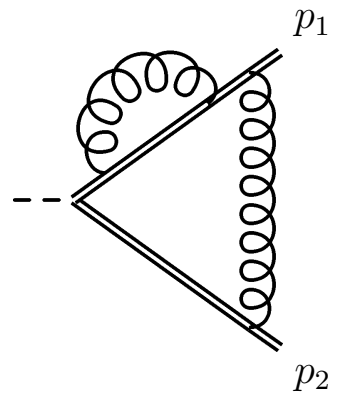

(d)

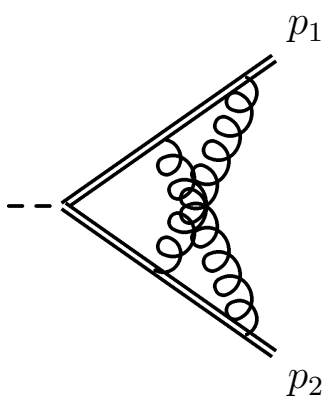

(b)

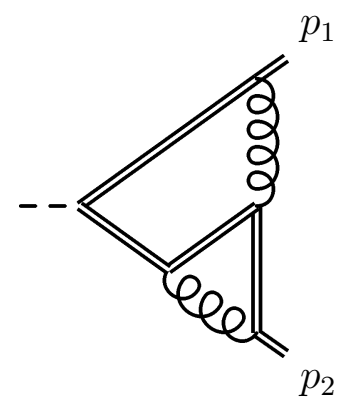

(c)

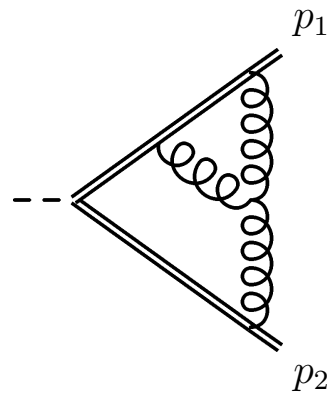

(e)

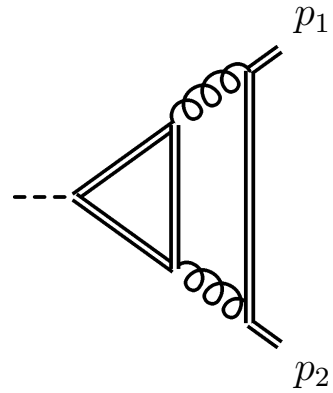

(f)

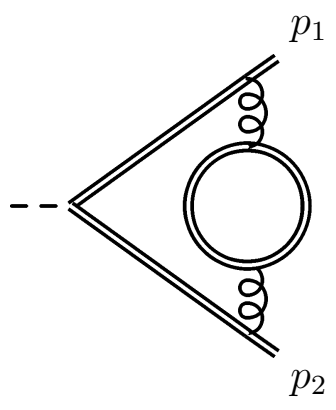

(g)

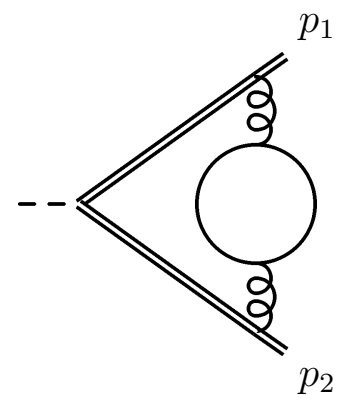

(h)

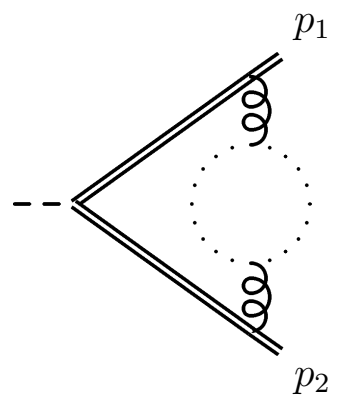

(i)

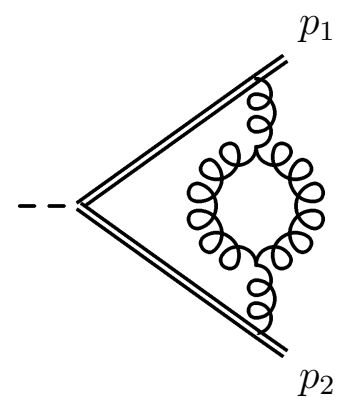

(j)

Figure 2: Two-loop vertex diagrams involved in the calculation of the form factors at order $\mathcal{O}\left(\alpha_{S}^{2}\right)$. The notation is as in Fig. 1. The single straight and dotted lines represent massless quarks and ghosts, respectively. The external quarks are on their mass-shell, $p_{1}^{2}=p_{2}^{2}=m^{2}$. Crossed diagrams are not drawn. 


\subsection{Scalar and Pseudoscalar Form Factors}

We consider the decay $h(q) \rightarrow Q\left(p_{1}\right)+\bar{Q}\left(p_{2}\right)$ and aim at computing the scalar and pseudoscalar parts of the $h Q \bar{Q}$ amplitude, for on-shell $Q, \bar{Q}$ and for arbitrary squared four-momentum $q^{2}=$ $s=\left(p_{1}+p_{2}\right)^{2}$, to second order in the QCD coupling $\alpha_{S}$ and to lowest order in the Yukawa couplings. For definiteness we work in QCD with $N_{f}$ massless flavors and one massive quark $Q$. The interaction (II) implies that the $h Q \bar{Q}$ amplitude $V$ is determined by two form factors:

$$
\begin{aligned}
V_{c_{1} c_{2}}\left(p_{1}, p_{2}\right) & =i \bar{u}_{c_{1}}\left(p_{1}\right) \Gamma_{c_{1} c_{2}}(q) v_{c_{2}}\left(p_{2}\right), \\
\Gamma_{c_{1} c_{2}}(q) & =-\frac{m}{v}\left(a_{Q} F_{S}(s)+i b_{Q} F_{P}(s) \gamma_{5}\right) \delta_{c_{1} c_{2}} .
\end{aligned}
$$

Here $F_{S}$ and $F_{P}$ denote the renormalized scalar and pseudoscalar form factors (whose tree-level values are equal to one), $m$ is the on-shell mass of $Q$, and $c_{1}, c_{2}$ are color labels. The expansion of these form factors in powers of $\alpha_{S} /(2 \pi)$, where $\alpha_{S}$ is the standard $\overline{\mathrm{MS}}$ coupling in $N_{f}+1$ flavor QCD defined at the renormalization scale $\mu$, reads

$$
\begin{aligned}
F_{i}\left(\epsilon, s, \frac{\mu^{2}}{m^{2}}\right)= & 1+\left(\frac{\alpha_{S}}{2 \pi}\right) F_{i}^{(1 l)}\left(\epsilon, s, \frac{\mu^{2}}{m^{2}}\right)+\left(\frac{\alpha_{S}}{2 \pi}\right)^{2} F_{i}^{(2 l)}\left(\epsilon, s, \frac{\mu^{2}}{m^{2}}\right) \\
& +\mathcal{O}\left(\left(\frac{\alpha_{S}}{2 \pi}\right)^{3}\right), \quad i=S, P .
\end{aligned}
$$

We regularize both the ultraviolet (UV) and infrared divergences using dimensional regularization in $D=4-2 \epsilon$ dimensions. We use a renormalization scheme where the wave function and the mass of $Q$ are defined in the on-shell scheme, see below. The UV-renormalized form factors (44) still depend on $\epsilon$ because they are infrared divergent.

The contributions to $\Gamma$ are, for the renormalization scheme employed here, the one-particleirreducible vertex functions shown in Figs. 1 and 2, and counterterm diagrams which are not displayed. We computed all of them in Feynman gauge.

The contribution to each form factor can be extracted from $V_{c_{1} c_{2}}$ by means of a suitable projection. In particular one has,

$$
\begin{aligned}
& F_{S}=-\frac{v}{2 m a_{Q}\left(s-4 m^{2}\right)} \operatorname{Tr}\left[I_{D}\left(\hat{p}_{1}+m\right) \Gamma\left(\hat{p}_{2}-m\right)\right], \\
& F_{P}=-\frac{i v}{2 m b_{Q} s} \operatorname{Tr}\left[\gamma_{5}\left(\hat{p}_{1}+m\right) \Gamma\left(\hat{p}_{2}-m\right)\right],
\end{aligned}
$$

where $\hat{p}=p_{\mu} \gamma^{\mu}$ and $I_{D}$ is the unit matrix in Dirac space.

In what follows $N_{f}$ denotes the number of light quarks running in the loops Fig. 2 (h), and $C_{F}=\left(N_{c}^{2}-1\right) /\left(2 N_{c}\right), C_{A}=N_{c}, T_{R}=1 / 2$, where $N_{c}$ is the number of colors. Notice that the scalar and pseudoscalar triangle diagrams analogous to Fig. 2 (f), with light quarks circulating in the triangles, give zero contribution because a trace over an odd number of $\gamma$ matrices is involved if the masses of these quarks are neglected in the triangle.

\subsection{Renormalization}

Before specifying our renormalization scheme, a remark on the prescription for the matrix $\gamma_{5}$

within dimensional regularization is in order. We use an anticommuting $\gamma_{5}$ in $D$ dimensions 
with $\gamma_{5}^{2}=1$. This is known to be an adequate prescription in the case of diagrams containing only open fermion lines, because it does not lead to spurious anomalies which must be canceled by performing an additional renormalization of the respective current, which in our case is the pseudoscalar current. As to the diagrams Figs. 1 and 2, an exception to this rule is the pseudoscalar triangle diagram Fig. 2 (f). However, this diagram is both UV- and IR-finite and may therefore be evaluated in 4 dimensions. It was computed already in [35].

We use renormalized perturbation theory with $\alpha_{S}=g_{s}^{2} /(4 \pi)$ being defined as the $\overline{\mathrm{MS}}$ coupling in $N_{f}+1$ flavor QCD, while we define the mass $m$ and the wave-function of the heavy quark $Q$ in the on-shell (OS) scheme.

For the renormalization of the $h Q \bar{Q}$ vertex function to two-loop order we need the coupling renormalization $Z_{g}^{\overline{\mathrm{MS}}}$ and the gluon wave function $Z_{3}^{\overline{\mathrm{MS}}}$ to one-loop, the latter one in the Feynman gauge. These renormalization constants are well-known and will not be reproduced here (c.f., e.g., [36]).

The renormalization constants of the heavy-quark mass and wave-function are defined in the OS-scheme. We need these constants to two-loop order; they were computed in [37,38]. Using the results of [38], we express the one- and two-loop contributions in terms of the renormalized $\overline{\mathrm{MS}}$ coupling $\alpha_{S}$. The quark wave function renormalization constant $Z_{2}^{\mathrm{O} S}$ expressed in this way may be found in [36]. Here we give only the mass renormalization constant $Z_{m}^{\mathrm{O} S}$ to two-loops $\left(m_{0}=Z_{m}^{\mathrm{O} S} m\right)$ : We have $Z_{m}^{\mathrm{O} S}=Z_{m, \mathrm{O} S}^{(1 l)}+Z_{m, \mathrm{O} S}^{(2 l)}$ where

$$
\begin{aligned}
Z_{m, \mathrm{O} S}^{(1 l)}= & -\frac{\alpha_{S}}{2 \pi} C(\epsilon)\left(\frac{\mu^{2}}{m^{2}}\right)^{\epsilon} \frac{C_{F}}{2} \frac{(3-2 \epsilon)}{\epsilon(1-2 \epsilon)}, \\
Z_{m, \mathrm{O} S}^{(2 l)}= & \left(\frac{\alpha_{S}}{2 \pi}\right)^{2} C^{2}(\epsilon)\left\{\left(\frac{\mu^{2}}{m^{2}}\right)^{2 \epsilon} \times\right. \\
& {\left[C_{F}\left(\frac{9}{32 \epsilon^{2}}+\frac{45}{64 \epsilon}+\frac{199}{128}-\frac{3}{4} \zeta_{3}+3 \zeta_{2} \ln (2)-\frac{15}{8} \zeta_{2}\right)\right.} \\
& +C_{A}\left(-\frac{11}{32 \epsilon^{2}}-\frac{91}{64 \epsilon}-\frac{605}{128}+\frac{3}{8} \zeta_{3}-\frac{3}{2} \zeta_{2} \ln (2)+\frac{1}{2} \zeta_{2}\right) \\
& +T_{R} N_{f}\left(\frac{1}{8 \epsilon^{2}}+\frac{7}{16 \epsilon}+\frac{45}{32}+\frac{1}{2} \zeta_{2}\right) \\
& \left.+T_{R}\left(\frac{1}{8 \epsilon^{2}}+\frac{7}{16 \epsilon}+\frac{69}{32}-\zeta_{2}\right)\right] \\
& +\left(\frac{\mu^{2}}{m^{2}}\right)^{\epsilon}\left[C_{F} C_{A}\left(\frac{33}{12 \epsilon^{2}}+\frac{11}{3 \epsilon}+\frac{22}{3}\right)\right. \\
& \left.\left.\quad-C_{F} T_{R}\left(N_{f}+1\right)\left(\frac{1}{\epsilon^{2}}+\frac{4}{3 \epsilon}+\frac{8}{3}\right)\right]\right\},
\end{aligned}
$$

and

$$
C(\epsilon)=(4 \pi)^{\epsilon} \Gamma(1+\epsilon)
$$

is an overall factor associated with each loop integral in $D$ dimensions. Here and in the following the Riemann zeta function of integer argument is denoted by $\zeta_{n}$. 
Further, we need the renormalization constant $Z_{1 \mathrm{~F}}$ for the $Q \bar{Q}$-gluon vertex to one loop. This constant is fixed by the Slavnov-Taylor identity $Z_{1 \mathrm{~F}}=Z_{g}^{\overline{\mathrm{MS}}} Z_{2}^{\mathrm{OS}} \sqrt{Z_{3}^{\overline{\mathrm{MS}}}}$.

Finally, we come to the overall renormalization of the $h Q \bar{Q}$ vertex function. Because of the interaction (11) the renormalization of the Yukawa couplings is fixed by the quark mass and wave function renormalization constants. Moreover, as stated above, in our prescription for $\gamma_{5}$ no additional counterterms are needed for the pseudoscalar vertex to order $\alpha_{S}^{2}$. Thus, the bare and renormalized $h Q \bar{Q}$ vertex functions are related by

$$
\Gamma=Z_{m}^{\mathrm{OS}} Z_{2}^{\mathrm{OS}} \Gamma_{B} .
$$

The counterterm diagram contributions to $\Gamma$ are, as far as their topological structure is concerned, identical to those given in [36], where the vector and axial vector parts of the $Z^{*} \rightarrow Q \bar{Q}$ amplitude to order $\alpha_{S}^{2}$ were computed, and will therefore not be exhibited here.

\section{Renormalized Form Factors for Spacelike $s<0$}

The renormalized form factors at the one- and two-loop level $F_{i}^{(1 l)}$ and $F_{i}^{(2 l)}(i=S, P)$ are computed with the technique that was applied in $[35,36,39]$ to the computation of the twoloop photon vertex in QED and to the two-loop vector and axial vector vertices in QCD, respectively. Performing the $D$-dimensional $\gamma$ algebra using FORM [40], we obtain the form factors expressed in terms of scalar integrals, which in the two-loop case amounts to several hundreds. They can be expressed as a combination of only 35 master integrals (MIs) by means of the by-now standard Laporta algorithm [41], which makes use of integration-by-parts identities [42], Lorentz invariance [43] and general symmetry relations. This reduction algorithm is performed exactly in $D=(4-2 \epsilon)$ dimensions [46]. Once the expression in terms of the MIs is found, one can expand the result in powers of $\epsilon$ around $\epsilon=0(D=4)$ and use the values of the MIs given in [44,45], which were evaluated with the differential equations method [47]. The expressions are given as a Laurent series in $\epsilon$ where both UV- and IR divergences, regularized by the same parameter $D$, show up as poles in $\epsilon$. After adding the counterterm diagrams, we obtain $F_{i}^{(1 l)}$ and $F_{i}^{(2 l)}$, and the remaining poles in $\epsilon$ are due to IR divergences.

Notice that the sum of the diagrams in Figs. 1 1 and 2 and of the counterterm diagrams has a non-trivial dependence on the renormalization scale $\mu$ : the counterterms with renormalization constants defined in the OS-scheme carry an overall factor $\left(\mu^{2} / \mathrm{m}^{2}\right)^{2 \epsilon}$; while those with renormalization constants defined in the in the $\overline{\mathrm{MS}}$-scheme carry just one factor $\left(\mu^{2} / \mathrm{m}^{2}\right)^{\epsilon}$, coming from the loop integration. Due to the mismatch between the $\epsilon$-dependence of the constants in the OS-scheme and of those in the $\overline{\mathrm{MS}}$-scheme, the $\epsilon$-expansion of the result generates terms proportional to $\ln \left(\mu^{2} / m^{2}\right)$ and $\ln ^{2}\left(\mu^{2} / m^{2}\right)$. In this section we give the one- and two-loop renormalized form factors choosing the renormalization scale at the value of the heavy quark mass, $\mu=m$. For the terms proportional to $\ln \left(\mu^{2} / m^{2}\right)$ and $\ln ^{2}\left(\mu^{2} / m^{2}\right)$ we refer the reader to Section 3.3 .

The one-loop form factors are presented below, including the term of order $\epsilon$ while the two-loop contributions are given to order $(\epsilon)^{0}$. They are expressed in terms of 1-dimensional harmonic polylogarithms $\mathrm{H}(\vec{a} ; x)$ up to weight $4[48,49]$, which are functions of the dimensionless 
variable $x$ defined by

$$
x=\frac{\sqrt{-s+4 m^{2}}-\sqrt{-s}}{\sqrt{-s+4 m^{2}}+\sqrt{-s}} .
$$

We give our results firstly in the kinematical region in which $q^{2}=s$ is spacelike $(0 \leq x \leq 1)$, where the form factors are real. In Section 4 we shall perform the analytical continuation to the physical region above threshold, $s>4 m^{2},-1<x \leq 0$, and explicitly decompose the form factors into real and imaginary parts.

\subsection{One-Loop Renormalized Form Factors}

The one-loop renormalized form factors are obtained by adding the contributions of diagram of Fig. 1 (b) and its counterterm.

\subsubsection{The Scalar Case}

By setting $\mu=m$ and defining

$$
F_{S}^{(1 l)}(s, \epsilon)=C(\epsilon) C_{F}\left(\frac{1}{\epsilon} a_{-1}+a_{0}+\epsilon a_{1}\right),
$$

one finds:

$$
\begin{aligned}
a_{-1}=\quad & -1-\frac{\left(1+x^{2}\right) H(0, x)}{1-x^{2}} ; \\
a_{0}=\quad & -\left(\frac{1-x^{2}-\zeta_{2}-x^{2} \zeta_{2}}{1-x^{2}}\right)+\frac{4 x H(0, x)}{1-x^{2}} \\
+ & \frac{2\left(1+x^{2}\right) H(-1,0, x)}{1-x^{2}}-\frac{\left(1+x^{2}\right) H(0,0, x)}{1-x^{2}} ; \\
a_{1}= & \frac{2\left(-1+x^{2}-2 x \zeta_{2}+\zeta_{3}+x^{2} \zeta_{3}\right)}{1-x^{2}} \\
& -\frac{2\left(1+x^{2}\right) \zeta_{2} H(-1, x)}{1-x^{2}} \\
+ & \frac{\left(-1+6 x-x^{2}+\zeta_{2}+x^{2} \zeta_{2}\right) H(0, x)}{1-x^{2}} \\
& -\frac{8 x H(-1,0, x)}{1-x^{2}+\frac{4 x H(0,0, x)}{1-x^{2}}} \\
& -\frac{4\left(1+x^{2}\right) H(-1,-1,0, x)}{1-x^{2}}+\frac{2\left(1+x^{2}\right) H(-1,0,0, x)}{1-x^{2}} \\
+ & \frac{2\left(1+x^{2}\right) H(0,-1,0, x)}{1-x^{2}}-\frac{\left(1+x^{2}\right) H(0,0,0, x)}{1-x^{2}} .
\end{aligned}
$$




\subsubsection{The Pseudoscalar Case}

Using an analogous expansion for the pseudoscalar form factor,

$$
F_{P}^{(1 l)}(s, \epsilon)=C(\epsilon) C_{F}\left(\frac{1}{\epsilon} \bar{a}_{-1}+\bar{a}_{0}+\epsilon \bar{a}_{1}\right),
$$

one finds:

$$
\begin{aligned}
\bar{a}_{-1}= & a_{-1} ; \\
\bar{a}_{0}=\quad & a_{0}-\frac{4 x H(0, x)}{1-x^{2}} ; \\
\bar{a}_{1}=\quad & \frac{-4\left(1+x^{2}\right) H(-1,-1,0, x)}{1-x^{2}}+\frac{2\left(1+x^{2}\right) H(-1,0,0, x)}{1-x^{2}} \\
& +\frac{2\left(1+x^{2}\right) H(0,-1,0, x)}{1-x^{2}}-\frac{\left(1+x^{2}\right) H(0,0,0, x)}{1-x^{2}} \\
& -\frac{2\left(1+x^{2}\right) H(-1, x) \zeta_{2}}{1-x^{2}} \\
& +\frac{H(0, x)\left(-1-2 x-x^{2}+\zeta_{2}+x^{2} \zeta_{2}\right)}{1-x^{2}} \\
& +\frac{2\left(-1+x^{2}+\zeta_{3}+x^{2} \zeta_{3}\right)}{1-x^{2}} .
\end{aligned}
$$

\subsection{Two-Loop Renormalized Form Factors}

The two-loop renormalized scalar and pseudoscalar form factors are obtained by adding the contributions of the diagrams in Fig. 2 (a)-(j), of the crossed ones, and of their counterterms. The pseudoscalar contribution of Fig. 22 (f) was already computed in [35] and it is added here for completeness.

\subsubsection{The Scalar Case}

By setting $\mu=m$ and defining

$$
\begin{aligned}
F_{S}^{(2 l)}(\epsilon, s)=C^{2}(\epsilon)\{ & C_{F}^{2}\left(\frac{b_{-2}}{\epsilon^{2}}+\frac{b_{-1}}{\epsilon}+b_{0}\right)+C_{F} C_{A}\left(\frac{c_{-2}}{\epsilon^{2}}+\frac{c_{-1}}{\epsilon}+c_{0}\right) \\
& +C_{F} T_{R} N_{f}\left(\frac{d_{-2}}{\epsilon^{2}}+\frac{d_{-1}}{\epsilon}+d_{0}\right)+C_{F} T_{R}\left(\frac{e_{-2}}{\epsilon^{2}}+\frac{e_{-1}}{\epsilon}+e_{0}\right) \\
& +\mathcal{O}(\epsilon)\},
\end{aligned}
$$

one finds: 


$$
\begin{aligned}
b_{-2}= & \frac{1}{2}+\frac{\left(1+x^{2}\right) H(0 ; x)}{1-x^{2}}+\frac{\left(1+x^{2}\right)^{2} H(0,0 ; x)}{\left(1-x^{2}\right)^{2}} ; \\
b_{-1}= & -\left(\frac{-1+x^{2}+\zeta_{2}+x^{2} \zeta_{2}}{1-x^{2}}\right) \\
& -\frac{\left(-1+4 x-4 x^{3}+x^{4}+\zeta_{2}+2 x^{2} \zeta_{2}+x^{4} \zeta_{2}\right) H(0 ; x)}{\left(1-x^{2}\right)^{2}} \\
& -\frac{2\left(1+x^{2}\right) H(-1,0 ; x)}{1-x^{2}}-\frac{\left(-1+8 x+8 x^{3}+x^{4}\right) H(0,0 ; x)}{\left(1-x^{2}\right)^{2}} \\
& -\frac{4\left(1+x^{2}\right)^{2} H(-1,0,0 ; x)}{\left(1-x^{2}\right)^{2}}-\frac{2\left(1+x^{2}\right)^{2} H(0,-1,0 ; x)}{\left(1-x^{2}\right)^{2}} \\
& +\frac{3\left(1+x^{2}\right)^{2} H(0,0,0 ; x)}{\left(1-x^{2}\right)^{2}} ;
\end{aligned}
$$

$$
\begin{aligned}
& b_{0}=\frac{1}{20(1-x)^{2}(1+x)^{3}}\left[145+145 x-290 x^{2}-290 x^{3}+145 x^{4}\right. \\
& +145 x^{5}+20 \zeta_{2}-760 x \zeta_{2}+1440 \ln (2) x \zeta_{2}+520 x^{2} \zeta_{2} \\
& -1440 \ln (2) x^{2} \zeta_{2}+720 x^{3} \zeta_{2}-1440 \ln (2) x^{3} \zeta_{2} \\
& -540 x^{4} \zeta_{2}+1440 \ln (2) x^{4} \zeta_{2}+40 x^{5} \zeta_{2}-118 \zeta_{2}^{2} \\
& +18 x \zeta_{2}^{2}-352 x^{2}{\zeta_{2}}^{2}+840 x^{3}{\zeta_{2}}^{2}+226 x^{4}{\zeta_{2}}^{2} \\
& +362 x^{5} \zeta_{2}^{2}-280 \zeta_{3}-960 x \zeta_{3}+200 x^{2} \zeta_{3} \\
& \left.+200 x^{3} \zeta_{3}-880 x^{4} \zeta_{3}-200 x^{5} \zeta_{3}\right] \\
& -\frac{\left(-5+49 x-53 x^{2}+x^{3}\right) \zeta_{2} H(-1 ; x)}{(1-x)(1+x)^{2}} \\
& -\frac{1}{(1-x)^{2}(1+x)^{3}}\left[-3+7 x+10 x^{2}-10 x^{3}-7 x^{4}+3 x^{5}\right. \\
& -2 \zeta_{2}-10 x \zeta_{2}+47 x^{2} \zeta_{2}-27 x^{3} \zeta_{2}-45 x^{4} \zeta_{2} \\
& +5 x^{5} \zeta_{2}+8 \zeta_{3}+24 x \zeta_{3}-56 x^{2} \zeta_{3}+56 x^{3} \zeta_{3} \\
& \left.-24 x^{4} \zeta_{3}-8 x^{5} \zeta_{3}\right] H(0 ; x) \\
& +\frac{4\left(1+x^{2}\right)^{2} \zeta_{3} H(1 ; x)}{\left(1-x^{2}\right)^{2}} \\
& +\frac{\left(15-42 x+42 x^{3}-15 x^{4}+4 \zeta_{2}+8 x^{2} \zeta_{2}+4 x^{4} \zeta_{2}\right) H(-1,0 ; x)}{\left(1-x^{2}\right)^{2}} \\
& -\frac{2\left(-7-7 x+16 x^{2}-20 x^{3}+5 x^{4}+5 x^{5}\right) \zeta_{2} H(0,-1 ; x)}{(1-x)^{2}(1+x)^{3}} \\
& +\frac{1}{(1-x)^{2}(1+x)^{3}}\left[3-12 x+36 x^{2}-22 x^{3}-47 x^{4}+26 x^{5}-3 \zeta_{2}\right.
\end{aligned}
$$




$$
\begin{aligned}
& \left.+x \zeta_{2}-12 x^{2} \zeta_{2}+32 x^{3} \zeta_{2}+9 x^{4} \zeta_{2}+13 x^{5} \zeta_{2}\right] H(0,0 ; x) \\
& +\frac{4}{(1-x)^{2}(1+x)^{3}}\left[-2+2 x+4 x^{2}-4 x^{3}-2 x^{4}+2 x^{5}+\zeta_{2}\right. \\
& \left.+5 x \zeta_{2}-18 x^{2} \zeta_{2}+22 x^{3} \zeta_{2}-3 x^{4} \zeta_{2}+x^{5} \zeta_{2}\right] H(1,0 ; x) \\
& +\frac{4\left(1+x^{2}\right) H(-1,-1,0 ; x)}{1-x^{2}} \\
& +\frac{\left(5+58 x-2 x^{2}+58 x^{3}+9 x^{4}\right) H(-1,0,0 ; x)}{\left(1-x^{2}\right)^{2}} \\
& +\frac{2\left(3-22 x+20 x^{2}-22 x^{3}+5 x^{4}\right) H(0,-1,0 ; x)}{\left(1-x^{2}\right)^{2}} \\
& -\frac{\left(-1+31 x+65 x^{2}-9 x^{3}+10 x^{5}\right) H(0,0,0 ; x)}{(1-x)^{2}(1+x)^{3}} \\
& -\frac{4\left(1-6 x+2 x^{2}-6 x^{3}+x^{4}\right) H(0,1,0 ; x)}{\left(1-x^{2}\right)^{2}} \\
& +\frac{2\left(5-16 x+6 x^{2}-16 x^{3}+5 x^{4}\right) H(1,0,0 ; x)}{\left(1-x^{2}\right)^{2}} \\
& +\frac{16\left(1+x^{2}\right)^{2} H(-1,-1,0,0 ; x)}{\left(1-x^{2}\right)^{2}}+\frac{8\left(1+x^{2}\right)^{2} H(-1,0,-1,0 ; x)}{\left(1-x^{2}\right)^{2}} \\
& -\frac{12\left(1+x^{2}\right)^{2} H(-1,0,0,0 ; x)}{\left(1-x^{2}\right)^{2}}+\frac{4\left(1+x^{2}\right)^{2} H(0,-1,-1,0 ; x)}{\left(1-x^{2}\right)^{2}} \\
& -\frac{2\left(3-5 x+36 x^{2}-16 x^{3}+15 x^{4}+7 x^{5}\right) H(0,-1,0,0 ; x)}{(1-x)^{2}(1+x)^{3}} \\
& -\frac{2\left(-1+3 x+10 x^{2}+10 x^{3}+7 x^{4}+11 x^{5}\right) H(0,0,-1,0 ; x)}{(1-x)^{2}(1+x)^{3}} \\
& +\frac{\left(7+7 x+16 x^{2}+52 x^{3}+27 x^{4}+27 x^{5}\right) H(0,0,0,0 ; x)}{(1-x)^{2}(1+x)^{3}} \\
& +\frac{4\left(-1+2 x^{2}+3 x^{4}\right) H(0,0,1,0 ; x)}{\left(1-x^{2}\right)^{2}} \\
& -\frac{4(-1+x) H(0,1,0,0 ; x)}{1+x}-\frac{8\left(1+x^{2}\right)^{2} H(1,0,-1,0 ; x)}{\left(1-x^{2}\right)^{2}} \\
& +\frac{8\left(1+3 x-8 x^{2}+12 x^{3}-x^{4}+x^{5}\right) H(1,0,0,0 ; x)}{(1-x)^{2}(1+x)^{3}} \\
& +\frac{8\left(1+x^{2}\right)^{2} H(1,0,1,0 ; x)}{\left(1-x^{2}\right)^{2}} \text {; } \\
& c_{-2}=\frac{11}{12}+\frac{11\left(1+x^{2}\right) H(0 ; x)}{12\left(1-x^{2}\right)} ;
\end{aligned}
$$




$$
\begin{aligned}
& c_{-1}=\frac{1}{36\left(1-x^{2}\right)^{2}}\left[-49+98 x^{2}-49 x^{4}+18 \zeta_{2}-72 x^{2} \zeta_{2}\right. \\
& \left.+54 x^{4} \zeta_{2}-18 \zeta_{3}-36 x^{2} \zeta_{3}-18 x^{4} \zeta_{3}\right] \\
& -\frac{\left(1+x^{2}\right)\left(67-67 x^{2}-18 \zeta_{2}+54 x^{2} \zeta_{2}\right) H(0 ; x)}{36\left(1-x^{2}\right)^{2}} \\
& +\frac{\left(1+x^{2}\right) H(-1,0 ; x)}{1-x^{2}}-\frac{2 x^{2} H(0,0 ; x)}{1-x^{2}} \\
& -\frac{\left(1+x^{2}\right) H(1,0 ; x)}{1-x^{2}}+\frac{\left(1+x^{2}\right)^{2} H(0,-1,0 ; x)}{\left(1-x^{2}\right)^{2}} \\
& -\frac{2\left(x^{2}+x^{4}\right) H(0,0,0 ; x)}{\left(1-x^{2}\right)^{2}}-\frac{\left(1+x^{2}\right)^{2} H(0,1,0 ; x)}{\left(1-x^{2}\right)^{2}} ; \\
& c_{0}=\frac{1}{540(1-x)^{2}(1+x)^{3}}\left[-4345-4345 x+8690 x^{2}+8690 x^{3}-4345 x^{4}\right. \\
& -4345 x^{5}+2730 \zeta_{2}+16860 x \zeta_{2}-19440 \ln (2) x \zeta_{2} \\
& -18900 x^{2} \zeta_{2}+19440 \ln (2) x^{2} \zeta_{2}-15840 x^{3} \zeta_{2} \\
& +19440 \ln (2) x^{3} \zeta_{2}+16170 x^{4} \zeta_{2}-19440 \ln (2) x^{4} \zeta_{2} \\
& -1020 x^{5} \zeta_{2}-81 \zeta_{2}{ }^{2}-81 x \zeta_{2}{ }^{2}-3132 x^{2} \zeta_{2}{ }^{2}-864 x^{3} \zeta_{2}{ }^{2} \\
& -1917 x^{4} \zeta_{2}^{2}-1917 x^{5} \zeta_{2}{ }^{2}+4410 \zeta_{3}-1530 x \zeta_{3} \\
& \left.+2700 x^{2} \zeta_{3}+2700 x^{3} \zeta_{3}-7110 x^{4} \zeta_{3}-1170 x^{5} \zeta_{3}\right] \\
& +\frac{1}{6\left(1-x^{2}\right)^{2}}\left[-25 \zeta_{2}+162 x \zeta_{2}-306 x^{2} \zeta_{2}+162 x^{3} \zeta_{2}\right. \\
& \left.+7 x^{4} \zeta_{2}+12 \zeta_{3}+24 x^{2} \zeta_{3}+12 x^{4} \zeta_{3}\right] H(-1 ; x) \\
& +\frac{1}{54(1-x)^{2}(1+x)^{3}}\left[-121+1133 x+1254 x^{2}-1254 x^{3}-1133 x^{4}\right. \\
& +121 x^{5}-99 \zeta_{2}+117 x \zeta_{2}+999 x^{2} \zeta_{2}+729 x^{3} \zeta_{2} \\
& -1764 x^{4} \zeta_{2}+18 x^{5} \zeta_{2}+351 \zeta_{3}+351 x \zeta_{3}+594 x^{2} \zeta_{3} \\
& \left.-702 x^{3} \zeta_{3}-405 x^{4} \zeta_{3}-405 x^{5} \zeta_{3}\right] H(0 ; x) \\
& -\frac{\left(1+x^{2}\right)\left(-\zeta_{2}+x^{2} \zeta_{2}+2 \zeta_{3}+2 x^{2} \zeta_{3}\right) H(1 ; x)}{\left(1-x^{2}\right)^{2}} \\
& -\frac{\left(-53+102 x-102 x^{3}+53 x^{4}-72 \zeta_{2}-72 x^{2} \zeta_{2}\right) H(-1,0 ; x)}{18\left(1-x^{2}\right)^{2}} \\
& +\frac{\left(-5-5 x+20 x^{2}-16 x^{3}+7 x^{4}+7 x^{5}\right) \zeta_{2} H(0,-1 ; x)}{(1-x)^{2}(1+x)^{3}} \\
& +\frac{1}{18(1-x)^{2}(1+x)^{3}}\left[-67+74 x+78 x^{2}-24 x^{3}-11 x^{4}-50 x^{5}\right. \\
& +18 \zeta_{2}+18 x \zeta_{2}+54 x^{2} \zeta_{2}+18 x^{3} \zeta_{2}+18 x^{4} \zeta_{2}
\end{aligned}
$$




$$
\begin{aligned}
& \left.+18 x^{5} \zeta_{2}\right] H(0,0 ; x) \\
& +\frac{\left(1+x^{2}\right)^{2} \zeta_{2} H(0,1 ; x)}{\left(1-x^{2}\right)^{2}} \\
& +\frac{2\left(1+x^{2}\right)\left(1-x^{2}-4 \zeta_{2}+2 x^{2} \zeta_{2}\right) H(1,0 ; x)}{\left(1-x^{2}\right)^{2}} \\
& -\frac{52\left(1+x^{2}\right) H(-1,-1,0 ; x)}{3\left(1-x^{2}\right)} \\
& +\frac{\left(61+175 x-35 x^{2}+79 x^{3}\right) H(-1,0,0 ; x)}{6(1-x)(1+x)^{2}} \\
& +\frac{6\left(1+x^{2}\right) H(-1,1,0 ; x)}{1-x^{2}} \\
& +\frac{2\left(1+10 x+25 x^{2}+34 x^{3}\right) H(0,-1,0 ; x)}{3(1-x)(1+x)^{2}} \\
& -\frac{\left(11-2 x-53 x^{2}-74 x^{3}+62 x^{4}\right) H(0,0,0 ; x)}{6(1-x)(1+x)^{3}} \\
& -\frac{4 x\left(1+x+2 x^{2}\right) H(0,1,0 ; x)}{(1-x)(1+x)^{2}} \\
& +\frac{6\left(1+x^{2}\right) H(1,-1,0 ; x)}{1-x^{2}}+\frac{4\left(-3+x^{2}\right) H(1,0,0 ; x)}{1-x^{2}} \\
& -\frac{2\left(1+x^{2}\right) H(1,1,0 ; x)}{1-x^{2}}-\frac{4\left(1+x^{2}\right)^{2} H(-1,0,-1,0 ; x)}{\left(1-x^{2}\right)^{2}} \\
& +\frac{2\left(3+4 x^{2}+x^{4}\right) H(-1,0,0,0 ; x)}{\left(1-x^{2}\right)^{2}}+\frac{4\left(1+x^{2}\right)^{2} H(-1,0,1,0 ; x)}{\left(1-x^{2}\right)^{2}} \\
& -\frac{10\left(1+x^{2}\right)^{2} H(0,-1,-1,0 ; x)}{\left(1-x^{2}\right)^{2}} \\
& +\frac{2\left(1+x+x^{2}+15 x^{3}+7 x^{4}+7 x^{5}\right) H(0,-1,0,0 ; x)}{(1-x)^{2}(1+x)^{3}} \\
& +\frac{6\left(1+x^{2}\right)^{2} H(0,-1,1,0 ; x)}{\left(1-x^{2}\right)^{2}} \\
& +\frac{2\left(1+x+22 x^{2}+2 x^{3}+11 x^{4}+11 x^{5}\right) H(0,0,-1,0 ; x)}{(1-x)^{2}(1+x)^{3}} \\
& -\frac{3 x^{2}\left(1+7 x+4 x^{2}+4 x^{3}\right) H(0,0,0,0 ; x)}{(1-x)^{2}(1+x)^{3}} \\
& -\frac{2\left(1+8 x^{2}+7 x^{4}\right) H(0,0,1,0 ; x)}{\left(1-x^{2}\right)^{2}}+\frac{6\left(1+x^{2}\right)^{2} H(0,1,-1,0 ; x)}{\left(1-x^{2}\right)^{2}} \\
& -\frac{2\left(3+3 x-8 x^{2}+16 x^{3}+x^{4}+x^{5}\right) H(0,1,0,0 ; x)}{(1-x)^{2}(1+x)^{3}}
\end{aligned}
$$




$$
\begin{aligned}
& -\frac{2\left(1+x^{2}\right)^{2} H(0,1,1,0 ; x)}{\left(1-x^{2}\right)^{2}}+\frac{4\left(1+x^{2}\right)^{2} H(1,0,-1,0 ; x)}{\left(1-x^{2}\right)^{2}} \\
& +\frac{2\left(-5-4 x^{2}+x^{4}\right) H(1,0,0,0 ; x)}{\left(1-x^{2}\right)^{2}}-\frac{4\left(1+x^{2}\right)^{2} H(1,0,1,0 ; x)}{\left(1-x^{2}\right)^{2}} \text {; } \\
& d_{-2}=-\frac{1}{3}-\frac{\left(1+x^{2}\right) H(0 ; x)}{3\left(1-x^{2}\right)} ; \\
& d_{-1}=\frac{5}{9}+\frac{5\left(1+x^{2}\right) H(0 ; x)}{9\left(1-x^{2}\right)} ; \\
& d_{0}=-\frac{-49+49 x^{2}-6 \zeta_{2}-72 x \zeta_{2}+66 x^{2} \zeta_{2}+36 \zeta_{3}+36 x^{2} \zeta_{3}}{27\left(1-x^{2}\right)} \\
& +\frac{4\left(1+x^{2}\right) \zeta_{2} H(-1 ; x)}{3\left(1-x^{2}\right)} \\
& +\frac{2\left(14-96 x+14 x^{2}+9 \zeta_{2}+9 x^{2} \zeta_{2}\right) H(0 ; x)}{27\left(1-x^{2}\right)} \\
& -\frac{4\left(5-12 x+5 x^{2}\right) H(-1,0 ; x)}{9\left(1-x^{2}\right)}+\frac{2\left(5-12 x+5 x^{2}\right) H(0,0 ; x)}{9\left(1-x^{2}\right)} \\
& +\frac{8\left(1+x^{2}\right) H(-1,-1,0 ; x)}{3\left(1-x^{2}\right)}-\frac{4\left(1+x^{2}\right) H(-1,0,0 ; x)}{3\left(1-x^{2}\right)} \\
& -\frac{4\left(1+x^{2}\right) H(0,-1,0 ; x)}{3\left(1-x^{2}\right)}+\frac{2\left(1+x^{2}\right) H(0,0,0 ; x)}{3\left(1-x^{2}\right)} ; \\
& e_{-2}=0 \\
& e_{-1}=0 \text {; } \\
& e_{0}=-\frac{1}{135(1-x)^{3}(1+x)^{4}}\left[-2035-1435 x+5505 x^{2}+4905 x^{3}\right. \\
& -4905 x^{4}-5505 x^{5}+1435 x^{6}+2035 x^{7}+810 \zeta_{2}+270 x \zeta_{2} \\
& -9810 x^{2} \zeta_{2}+9450 x^{3} \zeta_{2}-810 x^{4} \zeta_{2}+5490 x^{5} \zeta_{2} \\
& -4590 x^{6} \zeta_{2}-810 x^{7} \zeta_{2}+432 x \zeta_{2}{ }^{2}+2160 x^{2} \zeta_{2}{ }^{2} \\
& +2592 x^{3} \zeta_{2}{ }^{2}+2592 x^{4} \zeta_{2}{ }^{2}+2160 x^{5} \zeta_{2}{ }^{2}+432 x^{6} \zeta_{2}{ }^{2} \\
& \left.+8640 x^{2} \zeta_{3}+8640 x^{3} \zeta_{3}-8640 x^{4} \zeta_{3}-8640 x^{5} \zeta_{3}\right] \\
& +\frac{2}{27\left(1-x^{2}\right)^{3}}\left[28+144 x+212 x^{2}-768 x^{3}+212 x^{4}+144 x^{5}+28 x^{6}\right. \\
& +9 \zeta_{2}-9 x^{2} \zeta_{2}-9 x^{4} \zeta_{2}+9 x^{6} \zeta_{2}-162 x \zeta_{3}-648 x^{2} \zeta_{3} \\
& \left.+756 x^{3} \zeta_{3}-648 x^{4} \zeta_{3}-162 x^{5} \zeta_{3}\right] H(0 ; x) \\
& -\frac{32 x H(-1,0 ; x)}{1-x^{2}} \\
& -\frac{2}{9(1-x)^{3}(1+x)^{4}}\left[-5-23 x+101 x^{2}+531 x^{3}-27 x^{4}\right.
\end{aligned}
$$




$$
\begin{aligned}
& -353 x^{5}-229 x^{6}+5 x^{7}+18 x \zeta_{2}+90 x^{2} \zeta_{2}+468 x^{3} \zeta_{2} \\
& \left.+468 x^{4} \zeta_{2}+90 x^{5} \zeta_{2}+18 x^{6} \zeta_{2}\right] H(0,0 ; x) \\
& +\frac{8 x H(0,1 ; x)}{1-x^{2}} \\
& +\frac{8 x\left(2+4 x+2 x^{2}+\zeta_{2}+6 x \zeta_{2}+x^{2} \zeta_{2}\right) H(1,0 ; x)}{(1-x)(1+x)^{3}} \\
& +\frac{128 x^{2} H(0,-1,0 ; x)}{\left(1-x^{2}\right)^{2}-\frac{2\left(-1+48 x^{2}+x^{4}\right) H(0,0,0 ; x)}{3\left(1-x^{2}\right)^{2}}} \\
& -\frac{64 x^{2} H(0,1,0 ; x)}{\left(1-x^{2}\right)^{2}+\frac{128 x^{2} H(1,0,0 ; x)}{\left(1-x^{2}\right)^{2}}-\frac{256 x^{3} H(0,0,-1,0 ; x)}{\left(1-x^{2}\right)^{3}}} \\
& -\frac{2 x(1+x) H(0,0,0,0 ; x)}{(1-x)^{3}-\frac{12 x(1+x) H(0,0,0,1 ; x)}{(1-x)(1+x)^{3}}} \\
& -\frac{8 x\left(1+6 x+x^{2}\right) H(0,0,1,0 ; x)}{\left(1-x^{2}\right)^{3}} \\
& +\frac{8 x\left(1+4 x-26 x^{2}+4 x^{3}+x^{4}\right) H(0,1,0,0 ; x)}{(1-x)(1+x)^{3}} \\
& +\frac{8 x\left(1+6 x+x^{2}\right) H(1,0,0,0 ; x)}{(1-x)}
\end{aligned}
$$

\subsubsection{The Pseudoscalar Case}

As done in the scalar case, by choosing $\mu=m$ and defining

$$
\begin{aligned}
F_{P}^{(2 l)}(\epsilon, s)=C^{2}(\epsilon)\{ & C_{F}^{2}\left(\frac{\bar{b}_{-2}}{\epsilon^{2}}+\frac{\bar{b}_{-1}}{\epsilon}+\bar{b}_{0}\right)+C_{F} C_{A}\left(\frac{\bar{c}_{-2}}{\epsilon^{2}}+\frac{\bar{c}_{-1}}{\epsilon}+\bar{c}_{0}\right) \\
& +C_{F} T_{R} N_{f}\left(\frac{\bar{d}_{-2}}{\epsilon^{2}}+\frac{\bar{d}_{-1}}{\epsilon}+\bar{d}_{0}\right)+C_{F} T_{R}\left(\frac{\bar{e}_{-2}}{\epsilon^{2}}+\frac{\bar{e}_{-1}}{\epsilon}+\bar{e}_{0}\right) \\
& +\mathcal{O}(\epsilon)\},
\end{aligned}
$$

one finds:

$$
\begin{aligned}
\bar{b}_{-2}= & b_{-2} ; \\
\bar{b}_{-1}= & b_{-1}+\frac{4 x H(0 ; x)}{1-x^{2}}+\frac{8 x\left(1+x^{2}\right) H(0,0 ; x)}{\left(1-x^{2}\right)^{2}} ; \\
\bar{b}_{0}= & \frac{4\left(1+x^{2}\right) H(-1,-1,0 ; x)}{1-x^{2}} \\
& +\frac{\left(5+14 x+46 x^{2}+14 x^{3}+9 x^{4}\right) H(-1,0,0 ; x)}{\left(1-x^{2}\right)^{2}}
\end{aligned}
$$




$$
\begin{aligned}
& +\frac{2\left(3-14 x-4 x^{2}-14 x^{3}+5 x^{4}\right) H(0,-1,0 ; x)}{\left(1-x^{2}\right)^{2}} \\
& +\frac{\left(1-9 x-9 x^{2}+71 x^{3}-16 x^{4}+10 x^{5}\right) H(0,0,0 ; x)}{(1-x)^{3}(1+x)^{2}} \\
& -\frac{4\left(1+x^{2}\right) H(0,1,0 ; x)}{(1+x)^{2}}+\frac{2\left(5-12 x-18 x^{2}-12 x^{3}+5 x^{4}\right) H(1,0,0 ; x)}{\left(1-x^{2}\right)^{2}} \\
& +\frac{16\left(1+x^{2}\right)^{2} H(-1,-1,0,0 ; x)}{\left(1-x^{2}\right)^{2}}+\frac{8\left(1+x^{2}\right)^{2} H(-1,0,-1,0 ; x)}{\left(1-x^{2}\right)^{2}} \\
& -\frac{12\left(1+x^{2}\right)^{2} H(-1,0,0,0 ; x)}{\left(1-x^{2}\right)^{2}}+\frac{4\left(1+x^{2}\right)^{2} H(0,-1,-1,0 ; x)}{\left(1-x^{2}\right)^{2}} \\
& +\frac{2\left(-3+11 x-12 x^{2}+8 x^{3}+x^{4}+7 x^{5}\right) H(0,-1,0,0 ; x)}{(1-x)^{3}(1+x)^{2}} \\
& +\frac{2\left(1-5 x-10 x^{2}+10 x^{3}-15 x^{4}+11 x^{5}\right) H(0,0,-1,0 ; x)}{(1-x)^{3}(1+x)^{2}} \\
& -\frac{\left(-7+7 x-24 x^{2}+44 x^{3}-27 x^{4}+27 x^{5}\right) H(0,0,0,0 ; x)}{(1-x)^{3}(1+x)^{2}} \\
& +\frac{4\left(-1+2 x^{2}+3 x^{4}\right) H(0,0,1,0 ; x)}{\left(1-x^{2}\right)^{2}} \\
& +\frac{4\left(1-4 x+2 x^{2}-4 x^{3}+x^{4}\right) H(0,1,0,0 ; x)}{(1-x)^{3}(1+x)} \\
& -\frac{8\left(1+x^{2}\right)^{2} H(1,0,-1,0 ; x)}{\left(1-x^{2}\right)^{2}} \\
& +\frac{8\left(1+2 x+2 x^{2}-2 x^{3}+x^{4}\right) H(1,0,0,0 ; x)}{\left(1-x^{2}\right)^{2}} \\
& +\frac{8\left(1+x^{2}\right)^{2} H(1,0,1,0 ; x)}{\left(1-x^{2}\right)^{2}} \\
& +\frac{\left(5-18 x+54 x^{2}-18 x^{3}+x^{4}\right) H(-1 ; x) \zeta_{2}}{\left(1-x^{2}\right)^{2}} \\
& +\frac{2\left(7-7 x+8 x^{2}+4 x^{3}-5 x^{4}+5 x^{5}\right) H(0,-1 ; x) \zeta_{2}}{(1-x)^{3}(1+x)^{2}} \\
& +\frac{4 H(1,0 ; x)\left(-2+2 x^{4}+\zeta_{2}+4 x \zeta_{2}+2 x^{2} \zeta_{2}-4 x^{3} \zeta_{2}+x^{4} \zeta_{2}\right)}{\left(1-x^{2}\right)^{2}} \\
& +\frac{H(-1,0 ; x)\left(15+2 x-2 x^{3}-15 x^{4}+4 \zeta_{2}+8 x^{2} \zeta_{2}+4 x^{4} \zeta_{2}\right)}{\left(1-x^{2}\right)^{2}} \\
& -\frac{1}{(1-x)^{3}(1+x)^{2}}\left[-3+14 x+6 x^{2}-8 x^{3}-35 x^{4}+26 x^{5}+3 \zeta_{2}\right. \\
& \left.-7 x \zeta_{2}+4 x^{2} \zeta_{2}+24 x^{3} \zeta_{2}-17 x^{4} \zeta_{2}+13 x^{5} \zeta_{2}\right] H(0,0 ; x)
\end{aligned}
$$




$$
\begin{aligned}
& +\frac{4\left(1+x^{2}\right)^{2} H(1 ; x) \zeta_{3}}{\left(1-x^{2}\right)^{2}} \\
& -\frac{1}{20(1-x)^{3}(1+x)^{2}}\left[-145+145 x+290 x^{2}-290 x^{3}-145 x^{4}+145 x^{5}\right. \\
& -20 \zeta_{2}+240 x \zeta_{2}-1600 x^{2} \zeta_{2}+1640 x^{3} \zeta_{2}-300 x^{4} \zeta_{2}+40 x^{5} \zeta_{2} \\
& -480 x \ln (2) \zeta_{2}+1440 x^{2} \ln (2) \zeta_{2}-1440 x^{3} \ln (2) \zeta_{2} \\
& +480 x^{4} \ln (2) \zeta_{2}+118 \zeta_{2}{ }^{2}-254 x \zeta_{2}{ }^{2}+48 x^{2} \zeta_{2}{ }^{2}+536 x^{3} \zeta_{2}{ }^{2} \\
& -498 x^{4} \zeta_{2}{ }^{2}+362 x^{5} \zeta_{2}{ }^{2}+280 \zeta_{3}+280 x^{2} \zeta_{3}-280 x^{3} \zeta_{3} \\
& \left.-80 x^{4} \zeta_{3}-200 x^{5} \zeta_{3}\right] \\
& +\frac{H(0 ; x)}{(1-x)^{3}(1+x)^{2}}\left[3-x-2 x^{2}-2 x^{3}-x^{4}+3 x^{5}+2 \zeta_{2}-6 x \zeta_{2}\right. \\
& -11 x^{2} \zeta_{2}+73 x^{3} \zeta_{2}-15 x^{4} \zeta_{2}+5 x^{5} \zeta_{2}-8 \zeta_{3}-8 x \zeta_{3} \\
& \left.+8 x^{2} \zeta_{3}+8 x^{3} \zeta_{3}-8 x^{4} \zeta_{3}-8 x^{5} \zeta_{3}\right] \text {; } \\
& \bar{c}_{-2}=c_{-2} \\
& \bar{c}_{-1}=c_{-1} \\
& \bar{c}_{0}=\frac{-52\left(1+x^{2}\right) H(-1,-1,0 ; x)}{3\left(1-x^{2}\right)} \\
& -\frac{\left(-61-6 x+66 x^{2}-6 x^{3}+79 x^{4}\right) H(-1,0,0 ; x)}{6\left(1-x^{2}\right)^{2}} \\
& +\frac{6\left(1+x^{2}\right) H(-1,1,0 ; x)}{1-x^{2}} \\
& -\frac{2\left(-1-15 x-15 x^{2}-15 x^{3}+34 x^{4}\right) H(0,-1,0 ; x)}{3\left(1-x^{2}\right)^{2}} \\
& -\frac{\left(11-35 x+45 x^{2}+189 x^{3}-128 x^{4}+62 x^{5}\right) H(0,0,0 ; x)}{6(1-x)^{3}(1+x)^{2}} \\
& -\frac{4 x\left(1+x+2 x^{2}\right) H(0,1,0 ; x)}{(1-x)(1+x)^{2}} \\
& +\frac{6\left(1+x^{2}\right) H(1,-1,0 ; x)}{1-x^{2}} \\
& -\frac{4\left(3-2 x-4 x^{2}-2 x^{3}+x^{4}\right) H(1,0,0 ; x)}{\left(1-x^{2}\right)^{2}} \\
& -\frac{2\left(1+x^{2}\right) H(1,1,0 ; x)}{1-x^{2}} \\
& -\frac{4\left(1+x^{2}\right)^{2} H(-1,0,-1,0 ; x)}{\left(1-x^{2}\right)^{2}}
\end{aligned}
$$




$$
\begin{aligned}
& +\frac{2\left(3+4 x^{2}+x^{4}\right) H(-1,0,0,0 ; x)}{\left(1-x^{2}\right)^{2}} \\
& +\frac{4\left(1+x^{2}\right)^{2} H(-1,0,1,0 ; x)}{\left(1-x^{2}\right)^{2}} \\
& -\frac{10\left(1+x^{2}\right)^{2} H(0,-1,-1,0 ; x)}{\left(1-x^{2}\right)^{2}} \\
& -\frac{2\left(-1+x-5 x^{2}+11 x^{3}-7 x^{4}+7 x^{5}\right) H(0,-1,0,0 ; x)}{(1-x)^{3}(1+x)^{2}} \\
& +\frac{6\left(1+x^{2}\right)^{2} H(0,-1,1,0 ; x)}{\left(1-x^{2}\right)^{2}} \\
& -\frac{2\left(-1+x-14 x^{2}+10 x^{3}-11 x^{4}+11 x^{5}\right) H(0,0,-1,0 ; x)}{(1-x)^{3}(1+x)^{2}} \\
& +\frac{x^{2}\left(-7+17 x-12 x^{2}+12 x^{3}\right) H(0,0,0,0 ; x)}{(1-x)^{3}(1+x)^{2}} \\
& -\frac{2\left(1+8 x^{2}+7 x^{4}\right) H(0,0,1,0 ; x)}{\left(1-x^{2}\right)^{2}} \\
& +\frac{6\left(1+x^{2}\right)^{2} H(0,1,-1,0 ; x)}{\left(1-x^{2}\right)^{2}} \\
& +\frac{2\left(-3+3 x+8 x^{3}-x^{4}+x^{5}\right) H(0,1,0,0 ; x)}{(1-x)^{3}(1+x)^{2}} \\
& +\frac{\left(1+x^{2}\right)^{2} H(0,1 ; x) \zeta_{2}}{\left(1-x^{2}\right)^{2}} \\
& -\frac{H(-1,0 ; x)\left(-53-54 x+54 x^{3}+53 x^{4}-72 \zeta_{2}-72 x^{2} \zeta_{2}\right)}{\left(1-x^{2}\right) H(1,0 ; x)\left(1-x^{2}-4 \zeta_{2}+2 x^{2} \zeta_{2}\right)} \\
& -\frac{2\left(1+x^{2}\right)^{2} H(0,1,1,0 ; x)}{\left(1-x^{2}\right)^{2}} \\
& +\frac{4\left(1+x^{2}\right)^{2} H(1,0,-1,0 ; x)}{\left(1-x^{2}\right)^{2}} \\
& \left.+x^{2}\right)^{2} H(1,0,1,0 ; x) \\
& +5 x^{2}
\end{aligned}
$$




$$
\begin{aligned}
& -\frac{H(0,0 ; x)}{18(1-x)^{3}(1+x)^{2}}\left[67-148 x+36 x^{2}-90 x^{3}+185 x^{4}-50 x^{5}-18 \zeta_{2}\right. \\
& \left.+18 x \zeta_{2}-126 x^{2} \zeta_{2}-54 x^{3} \zeta_{2}-18 x^{4} \zeta_{2}+18 x^{5} \zeta_{2}\right] \\
& -\frac{\left(1+x^{2}\right) H(1 ; x)\left(-\zeta_{2}+x^{2} \zeta_{2}+2 \zeta_{3}+2 x^{2} \zeta_{3}\right)}{\left(1-x^{2}\right)^{2}} \\
& +\frac{H(-1 ; x)}{6\left(1-x^{2}\right)^{2}}\left[-25 \zeta_{2}+54 x \zeta_{2}-162 x^{2} \zeta_{2}+54 x^{3} \zeta_{2}+7 x^{4} \zeta_{2}\right. \\
& \left.+12 \zeta_{3}+24 x^{2} \zeta_{3}+12 x^{4} \zeta_{3}\right] \\
& -\frac{1}{540(1-x)^{3}(1+x)^{2}}\left[4345-4345 x-8690 x^{2}+8690 x^{3}+4345 x^{4}\right. \\
& -4345 x^{5}-2730 \zeta_{2}-1320 x \zeta_{2}+25200 x^{2} \zeta_{2}-23580 x^{3} \zeta_{2} \\
& +3450 x^{4} \zeta_{2}-1020 x^{5} \zeta_{2}+6480 x \ln (2) \zeta_{2}-19440 x^{2} \ln (2) \zeta_{2} \\
& +19440 x^{3} \ln (2) \zeta_{2}-6480 x^{4} \ln (2) \zeta_{2}+81 \zeta_{2}{ }^{2}-81 x \zeta_{2}{ }^{2} \\
& -108 x^{2} \zeta_{2}^{2}-4104 x^{3}{\zeta_{2}}^{2}+1917 x^{4} \zeta_{2}^{2}-1917 x^{5} \zeta_{2}{ }^{2}-4410 \zeta_{3} \\
& \left.+4950 x \zeta_{3}-2700 x^{2} \zeta_{3}+2700 x^{3} \zeta_{3}+630 x^{4} \zeta_{3}-1170 x^{5} \zeta_{3}\right] \\
& -\frac{H(0 ; x)}{54(1-x)^{3}(1+x)^{2}}\left[121-283 x+162 x^{2}+162 x^{3}-283 x^{4}+121 x^{5}\right. \\
& +99 \zeta_{2}-315 x \zeta_{2}-135 x^{2} \zeta_{2}+2241 x^{3} \zeta_{2}-612 x^{4} \zeta_{2} \\
& +18 x^{5} \zeta_{2}-351 \zeta_{3}+351 x \zeta_{3}-162 x^{2} \zeta_{3}-270 x^{3} \zeta_{3} \\
& \left.+405 x^{4} \zeta_{3}-405 x^{5} \zeta_{3}\right] \\
& \bar{d}_{-2}=d_{-2} \\
& \bar{d}_{-1}=d_{-1} \\
& \bar{d}_{0}=\frac{-20\left(1+x^{2}\right) H(-1,0 ; x)}{9\left(1-x^{2}\right)}+\frac{10\left(1+x^{2}\right) H(0,0 ; x)}{9\left(1-x^{2}\right)} \\
& +\frac{8\left(1+x^{2}\right) H(-1,-1,0 ; x)}{3\left(1-x^{2}\right)}-\frac{4\left(1+x^{2}\right) H(-1,0,0 ; x)}{3\left(1-x^{2}\right)} \\
& -\frac{4\left(1+x^{2}\right) H(0,-1,0 ; x)}{3\left(1-x^{2}\right)}+\frac{2\left(1+x^{2}\right) H(0,0,0 ; x)}{3\left(1-x^{2}\right)} \\
& +\frac{4\left(1+x^{2}\right) H(-1 ; x) \zeta_{2}}{3\left(1-x^{2}\right)}+\frac{2\left(1+x^{2}\right) H(0 ; x)\left(14+9 \zeta_{2}\right)}{27\left(1-x^{2}\right)} \\
& -\frac{-49+49 x^{2}-6 \zeta_{2}+66 x^{2} \zeta_{2}+36 \zeta_{3}+36 x^{2} \zeta_{3}}{27\left(1-x^{2}\right)} ;
\end{aligned}
$$

$\bar{e}_{-2}=e_{-2}=0 ;$ 


$$
\begin{aligned}
& \bar{e}_{-1}=e_{-1}=0 ; \\
& \bar{e}_{0}=\frac{2\left(1-2 x+26 x^{2}-2 x^{3}+x^{4}\right) H(0,0,0 ; x)}{3(1-x)^{3}(1+x)}-\frac{2 x H(0,0,0,0 ; x)}{1-x^{2}} \\
& -\frac{12 x H(0,0,0,1 ; x)}{1-x^{2}}-\frac{8 x H(0,0,1,0 ; x)}{1-x^{2}}+\frac{8 x H(0,1,0,0 ; x)}{1-x^{2}} \\
& +\frac{8 x H(1,0,0,0 ; x)}{1-x^{2}}+\frac{8 x H(1,0 ; x) \zeta_{2}}{1-x^{2}} \\
& +\frac{2 H(0,0 ; x)}{9(1-x)^{2}(1+x)^{4}}\left[5+22 x+59 x^{2}+116 x^{3}+59 x^{4}+22 x^{5}+5 x^{6}\right. \\
& \left.-18 x \zeta_{2}-36 x^{2} \zeta_{2}+36 x^{4} \zeta_{2}+18 x^{5} \zeta_{2}\right] \\
& +\frac{2 H(0 ; x)}{27\left(1-x^{2}\right)^{3}}\left[28+24 x-76 x^{2}+48 x^{3}-76 x^{4}+24 x^{5}+28 x^{6}+9 \zeta_{2}\right. \\
& +207 x^{2} \zeta_{2}+432 x^{3} \zeta_{2}+207 x^{4} \zeta_{2}+9 x^{6} \zeta_{2}-162 x \zeta_{3} \\
& \left.+324 x^{3} \zeta_{3}-162 x^{5} \zeta_{3}\right] \\
& +\frac{1}{135(1-x)^{2}(1+x)^{4}}\left[2035+4190 x-2035 x^{2}-8380 x^{3}-2035 x^{4}\right. \\
& +4190 x^{5}+2035 x^{6}-810 \zeta_{2}-1080 x \zeta_{2}+4410 x^{2} \zeta_{2}+12240 x^{3} \zeta_{2} \\
& +4410 x^{4} \zeta_{2}-1080 x^{5} \zeta_{2}-810 x^{6} \zeta_{2}-432 x \zeta_{2}{ }^{2}-864 x^{2} \zeta_{2}{ }^{2} \\
& \left.+864 x^{4}{\zeta_{2}}^{2}+432 x^{5} \zeta_{2}^{2}\right] \text {. }
\end{aligned}
$$

\subsection{Form Factors for $\mu \neq m$}

At the one-loop level, the dependence of the form factors on the renormalization scale $\mu$ appears in an overall factor $\left(\mu^{2} / m^{2}\right)^{\epsilon}$ :

$$
F_{i}^{(1 l)}\left(\epsilon, s, \frac{\mu^{2}}{m^{2}}\right)=C(\epsilon)\left(\frac{\mu^{2}}{m^{2}}\right)^{\epsilon} F_{i}^{(1 l)}(\epsilon, s), \quad(i=S, P)
$$

with the expression of $F_{S}^{(1 l)}(\epsilon, s)$ and $F_{P}^{(1 l)}(\epsilon, s)$ given in Eq. (12) and Eq. (16) respectively.

At the two-loop level, the coupling constant renormalization generates logarithms of the ratio $\left(\mu^{2} / m^{2}\right)$. Factoring an overall $\left(\mu^{2} / m^{2}\right)^{2 \epsilon}$, one has [36]:

$$
\begin{aligned}
F_{i}^{(2 l)}\left(\epsilon, s, \frac{\mu^{2}}{m^{2}}\right)= & \left(\frac{\alpha_{S}}{2 \pi}\right)^{2} C^{2}(\epsilon)\left(\frac{\mu^{2}}{m^{2}}\right)^{2 \epsilon} \\
& \times\left\{F_{i}^{(2 l)}(\epsilon, s)-\frac{\beta_{0}}{\epsilon}\left[\left(\frac{\mu^{2}}{m^{2}}\right)^{-\epsilon}-1\right] F_{i}^{(1 l)}(\epsilon, s)\right\} \\
= & \left(\frac{\alpha_{S}}{2 \pi}\right)^{2} C^{2}(\epsilon)\left(\frac{\mu^{2}}{m^{2}}\right)^{2 \epsilon}\left\{F_{i}^{(2 l)}(\epsilon, s)+\beta_{0} F_{i}^{(1 l)}(\epsilon, s) \ln \left(\frac{\mu^{2}}{m^{2}}\right)\right.
\end{aligned}
$$




$$
\left.-\epsilon \frac{\beta_{0}}{2} F_{i}^{(1 l)}(\epsilon, s) \ln ^{2}\left(\frac{\mu^{2}}{m^{2}}\right)\right\} \quad(i=S, P)
$$

where the functions $F_{i}^{(2 l)}(\epsilon, s)$ are given in Eq. (20) and Eq. (33), and $\beta_{0}$ is the first coefficient of the QCD $\beta$-function,

$$
\beta_{0}=\frac{11 C_{A}-4 T_{R}\left(N_{f}+1\right)}{6}
$$

\section{Analytic Continuation above Threshold}

For application to Higgs decay, the form factors must be analytically continued into the timelike region, above the $Q \bar{Q}$ threshold, $s>4 m^{2}$, where they develop imaginary parts. That can be made by the substitution $s \rightarrow s+i \epsilon$, which implies

$$
x \rightarrow-y+i \epsilon,
$$

where

$$
y=\frac{\sqrt{s}-\sqrt{s-4 m^{2}}}{\sqrt{s}+\sqrt{s-4 m^{2}}},
$$

with $y=1$ at $s=4 m^{2}$ and $y \rightarrow 0$ as $s \rightarrow \infty$.

The real and imaginary parts of the form factors are defined through the relations:

$$
F_{i}(\epsilon, s+i \epsilon)=\Re F_{i}(\epsilon, s)+i \pi \Im F_{i}(\epsilon, s) \quad(i=S, P) .
$$

In the following two sections we will give the real and imaginary parts of the one- and two-loop analytically continued form factors for $\mu=m$. The renormalization scale dependence follows from the pattern outlined in Section 3.3.

\subsection{One-Loop Form Factors above Threshold}

In this section we give the analytic continuation of the coefficients $a_{i}$, corresponding to the scalar form factor defined in Eq. (12), and $\bar{a}_{i}$, corresponding to the pseudoscalar one defined in Eq. (16).

\subsubsection{The Scalar Case}

$$
\begin{aligned}
\Re a_{-1}= & -1-\frac{\left(1+y^{2}\right) H(0 ; y)}{1-y^{2}} ; \\
\Re a_{0}= & -\left(\frac{1-y^{2}-4 \zeta_{2}-4 y^{2} \zeta_{2}}{1-y^{2}}\right)-\frac{4 y H(0 ; y)}{1-y^{2}} \\
& -\frac{\left(1+y^{2}\right) H(0,0 ; y)}{1-y^{2}}-\frac{2\left(1+y^{2}\right) H(1,0 ; y)}{1-y^{2}} ; \\
\Re a_{1}= & \frac{2\left(-1+y^{2}+8 y \zeta_{2}+\zeta_{3}+y^{2} \zeta_{3}\right)}{1-y^{2}}
\end{aligned}
$$




$$
\begin{aligned}
+ & \frac{\left(-1-6 y-y^{2}+4 \zeta_{2}+4 y^{2} \zeta_{2}\right) H(0 ; y)}{1-y^{2}} \\
+ & \frac{8\left(1+y^{2}\right) \zeta_{2} H(1 ; y)}{1-y^{2}}-\frac{4 y H(0,0 ; y)}{1-y^{2}}-\frac{8 y H(1,0 ; y)}{1-y^{2}} \\
- & \frac{\left(1+y^{2}\right) H(0,0,0 ; y)}{1-y^{2}}-\frac{2\left(1+y^{2}\right) H(0,1,0 ; y)}{1-y^{2}} \\
- & \frac{2\left(1+y^{2}\right) H(1,0,0 ; y)}{1-y^{2}}-\frac{4\left(1+y^{2}\right) H(1,1,0 ; y)}{1-y^{2}} \\
\Im a_{-1}= & -\frac{1+y^{2}}{1-y^{2}} ; \\
\Im a_{0}= & \frac{-4 y}{1-y^{2}}-\frac{\left(1+y^{2}\right) H(0 ; y)}{1-y^{2}}-\frac{2\left(1+y^{2}\right) H(1 ; y)}{1-y^{2}} ; \\
\Im a_{1}= & -\frac{1+6 y+y^{2}-2 \zeta_{2}-2 y^{2} \zeta_{2}}{1-y^{2}} \\
& -\frac{4 y H(0 ; y)}{1-y^{2}}-\frac{8 y H(1 ; y)}{1-y^{2}}-\frac{\left(1+y^{2}\right) H(0,0 ; y)}{1-y^{2}} \\
& -\frac{2\left(1+y^{2}\right) H(0,1 ; y)}{1-y^{2}}-\frac{2\left(1+y^{2}\right) H(1,0 ; y)}{1-y^{2}} \\
& -\frac{4\left(1+y^{2}\right) H(1,1 ; y)}{1-y^{2}} .
\end{aligned}
$$

\subsubsection{The Pseudoscalar Case}

$\Re \bar{a}_{-1}=\Re a_{-1} ;$

$$
\begin{aligned}
\Re \bar{a}_{0}= & -\frac{\left(1+y^{2}\right) H(0,0 ; y)}{1-y^{2}}-\frac{2\left(1+y^{2}\right) H(1,0 ; y)}{1-y^{2}} \\
& -\frac{1-y^{2}-4 \zeta_{2}-4 y^{2} \zeta_{2}}{1-y^{2}} ; \\
\Re \bar{a}_{1}=\quad & -\frac{\left(1+y^{2}\right) H(0,0,0 ; y)}{1-y^{2}}-\frac{2\left(1+y^{2}\right) H(0,1,0 ; y)}{1-y^{2}} \\
& -\frac{2\left(1+y^{2}\right) H(1,0,0 ; y)}{1-y^{2}}-\frac{4\left(1+y^{2}\right) H(1,1,0 ; y)}{1-y^{2}} \\
& +\frac{8\left(1+y^{2}\right) H(1 ; y) \zeta_{2}}{1-y^{2}} \\
& +\frac{H(0 ; y)\left(-1+2 y-y^{2}+4 \zeta_{2}+4 y^{2} \zeta_{2}\right)}{1-y^{2}} \\
& +\frac{2\left(-1+y^{2}+\zeta_{3}+y^{2} \zeta_{3}\right)}{1-y^{2}} .
\end{aligned}
$$




$$
\begin{aligned}
\Im \bar{a}_{-1}= & \Im a_{-1} ; \\
\Im \bar{a}_{0}= & -\frac{\left(1+y^{2}\right) H(0 ; y)}{1-y^{2}}-\frac{2\left(1+y^{2}\right) H(1 ; y)}{1-y^{2}} ; \\
\Im \bar{a}_{1}=\quad & -\frac{\left(1+y^{2}\right) H(0,0 ; y)}{1-y^{2}}-\frac{2\left(1+y^{2}\right) H(0,1 ; y)}{1-y^{2}} \\
& -\frac{2\left(1+y^{2}\right) H(1,0 ; y)}{1-y^{2}}-\frac{4\left(1+y^{2}\right) H(1,1 ; y)}{1-y^{2}} \\
& -\frac{1-2 y+y^{2}-2 \zeta_{2}-2 y^{2} \zeta_{2}}{1-y^{2}} .
\end{aligned}
$$

Our results Eqs. (53), (54) and Eqs. (59), (60) in the scalar and pseudoscalar cases, agree with those of [4] and [7], respectively.

\subsection{Two-Loop Form Factors above Threshold}

In this section we give the analytic continuation of the coefficients $b_{i}, c_{i}, d_{i}, e_{i}$, of the scalar form factor defined in Eq. (201) and $\bar{b}_{i}, \bar{c}_{i}, \bar{d}_{i}, \bar{e}_{i}$ of the pseudoscalar one, defined in Eq. (333).

\subsubsection{The Scalar Case}

$$
\begin{aligned}
\Re b_{-2}= & \frac{1-2 y^{2}+y^{4}-6 \zeta_{2}-12 y^{2} \zeta_{2}-6 y^{4} \zeta_{2}}{2\left(1-y^{2}\right)^{2}} \\
& +\frac{\left(1+y^{2}\right) H(0 ; y)}{1-y^{2}}+\frac{\left(1+y^{2}\right)^{2} H(0,0 ; y)}{\left(1-y^{2}\right)^{2}} ; \\
\Re b_{-1}= & \frac{1-2 y^{2}+y^{4}-4 \zeta_{2}-24 y \zeta_{2}-24 y^{3} \zeta_{2}+4 y^{4} \zeta_{2}}{\left(1-y^{2}\right)^{2}} \\
& -\frac{\left(-1-4 y+4 y^{3}+y^{4}+10 \zeta_{2}+20 y^{2} \zeta_{2}+10 y^{4} \zeta_{2}\right) H(0 ; y)}{\left(1-y^{2}\right)^{2}} \\
& -\frac{12\left(1+y^{2}\right)^{2} \zeta_{2} H(1 ; y)}{\left(1-y^{2}\right)^{2}}-\frac{\left(-1-8 y-8 y^{3}+y^{4}\right) H(0,0 ; y)}{\left(1-y^{2}\right)^{2}} \\
& +\frac{2\left(1+y^{2}\right) H(1,0 ; y)}{1-y^{2}}+\frac{3\left(1+y^{2}\right)^{2} H(0,0,0 ; y)}{\left(1-y^{2}\right)^{2}} \\
& +\frac{2\left(1+y^{2}\right)^{2} H(0,1,0 ; y)}{\left(1-y^{2}\right)^{2}}+\frac{4\left(1+y^{2}\right)^{2} H(1,0,0 ; y)}{\left(1-y^{2}\right)^{2}} ; \\
\Re b_{0}= & -\frac{1}{20(1-y)^{3}(1+y)^{2}}\left[-145+145 y+290 y^{2}-290 y^{3}-145 y^{4}\right. \\
+ & 145 y^{5}+160 \zeta_{2}-40 y \zeta_{2}+1440 \ln (2) y \zeta_{2}+1640 y^{2} \zeta_{2}
\end{aligned}
$$




$$
\begin{aligned}
& +1440 \ln (2) y^{2} \zeta_{2}+2040 y^{3} \zeta_{2}-1440 \ln (2) y^{3} \zeta_{2} \\
& -2280 y^{4} \zeta_{2}-1440 \ln (2) y^{4} \zeta_{2}-1520 y^{5} \zeta_{2}-272 \zeta_{2}^{2} \\
& +168 y \zeta_{2}^{2}-848 y^{2}{\zeta_{2}}^{2}+480 y^{3}{\zeta_{2}}^{2}-496 y^{4} \zeta_{2}^{2} \\
& +392 y^{5} \zeta_{2}^{2}+280 \zeta_{3}-960 y \zeta_{3}-200 y^{2} \zeta_{3}+200 y^{3} \zeta_{3} \\
& \left.+880 y^{4} \zeta_{3}-200 y^{5} \zeta_{3}\right] \\
& +\frac{2}{\left(1-y^{2}\right)^{2}}\left[15 \zeta_{2}+48 y \zeta_{2}+18 y^{2} \zeta_{2}+48 y^{3} \zeta_{2}+15 y^{4} \zeta_{2}\right. \\
& \left.-2 \zeta_{3}-4 y^{2} \zeta_{3}-2 y^{4} \zeta_{3}\right] H(-1 ; y) \\
& -\frac{1}{(1-y)^{3}(1+y)^{2}}\left[-3-7 y+10 y^{2}+10 y^{3}-7 y^{4}-3 y^{5}+\zeta_{2}\right. \\
& +103 y \zeta_{2}-148 y^{2} \zeta_{2}-45 y^{4} \zeta_{2}+25 y^{5} \zeta_{2}+8 \zeta_{3} \\
& \left.-24 y \zeta_{3}-56 y^{2} \zeta_{3}-56 y^{3} \zeta_{3}-24 y^{4} \zeta_{3}+8 y^{5} \zeta_{3}\right] H(0 ; y) \\
& +\frac{2\left(5-114 y-54 y^{2}-114 y^{3}+13 y^{4}\right) \zeta_{2} H(1 ; y)}{\left(1-y^{2}\right)^{2}} \\
& -\frac{4}{(1-y)^{3}(1+y)^{2}}\left[-2-2 y+4 y^{2}+4 y^{3}-2 y^{4}-2 y^{5}\right. \\
& -5 \zeta_{2}+13 y \zeta_{2}+30 y^{2} \zeta_{2}+50 y^{3} \zeta_{2}+3 y^{4} \zeta_{2} \\
& \left.+5 y^{5} \zeta_{2}\right] H(-1,0 ; y) \\
& +\frac{12(1+y) \zeta_{2} H(0,-1 ; y)}{1-y} \\
& +\frac{1}{(1-y)^{3}(1+y)^{2}}\left[3+12 y+36 y^{2}+22 y^{3}-47 y^{4}-26 y^{5}\right. \\
& -24 \zeta_{2}+20 y \zeta_{2}-60 y^{2} \zeta_{2}+124 y^{3} \zeta_{2}-72 y^{4} \zeta_{2} \\
& \left.+68 y^{5} \zeta_{2}\right] H(0,0 ; y) \\
& +\frac{8\left(-4-2 y-23 y^{2}-7 y^{3}-10 y^{4}+4 y^{5}\right) \zeta_{2} H(0,1 ; y)}{(1-y)^{3}(1+y)^{2}} \\
& -\frac{1}{\left(1-y^{2}\right)^{2}}\left[15+42 y-42 y^{3}-15 y^{4}+40 \zeta_{2}+80 y^{2} \zeta_{2}\right. \\
& \left.+40 y^{4} \zeta_{2}\right] H(1,0 ; y) \\
& -\frac{48\left(1+y^{2}\right)^{2} \zeta_{2} H(1,1 ; y)}{\left(1-y^{2}\right)^{2}} \\
& -\frac{2\left(5+16 y+6 y^{2}+16 y^{3}+5 y^{4}\right) H(-1,0,0 ; y)}{\left(1-y^{2}\right)^{2}} \\
& +\frac{4\left(1+6 y+2 y^{2}+6 y^{3}+y^{4}\right) H(0,-1,0 ; y)}{\left(1-y^{2}\right)^{2}}
\end{aligned}
$$




$$
\begin{aligned}
& +\frac{\left(1+31 y-65 y^{2}-9 y^{3}+10 y^{5}\right) H(0,0,0 ; y)}{(1-y)^{3}(1+y)^{2}} \\
& -\frac{2\left(3+22 y+20 y^{2}+22 y^{3}+5 y^{4}\right) H(0,1,0 ; y)}{\left(1-y^{2}\right)^{2}} \\
& -\frac{\left(5-58 y-2 y^{2}-58 y^{3}+9 y^{4}\right) H(1,0,0 ; y)}{\left(1-y^{2}\right)^{2}} \\
& +\frac{4\left(1+y^{2}\right) H(1,1,0 ; y)}{1-y^{2}}+\frac{8\left(1+y^{2}\right)^{2} H(-1,0,-1,0 ; y)}{\left(1-y^{2}\right)^{2}} \\
& +\frac{8\left(-1+3 y+8 y^{2}+12 y^{3}+y^{4}+y^{5}\right) H(-1,0,0,0 ; y)}{(1-y)^{3}(1+y)^{2}} \\
& -\frac{8\left(1+y^{2}\right)^{2} H(-1,0,1,0 ; y)}{\left(1-y^{2}\right)^{2}-\frac{4(1+y) H(0,-1,0,0 ; y)}{\left(1-y^{2}\right)^{2}}} \\
& -\frac{4\left(-1+2 y^{2}+3 y^{4}\right) H(0,0,-1,0 ; y)}{(1-y)^{3}(1+y)^{2}} \\
& -\frac{\left(-7+7 y-16 y^{2}+52 y^{3}-27 y^{4}+27 y^{5}\right) H(0,0,0,0 ; y)}{(1-y)^{3}(1+y)^{2}} \\
& -\frac{2\left(1+3 y-10 y^{2}+10 y^{3}-7 y^{4}+11 y^{5}\right) H(0,0,1,0 ; y)}{\left(1-y^{2}\right)^{2}} \\
& -\frac{2\left(-3-5 y-36 y^{2}-16 y^{3}-15 y^{4}+7 y^{5}\right) H(0,1,0,0 ; y)}{(1-y)^{3}(1+y)^{2}} \\
& +\frac{4\left(1+y^{2}\right)^{2} H(0,1,1,0 ; y)}{\left(1-y^{2}\right)^{2}}+\frac{12\left(1+y^{2}\right)^{2} H(1,0,0,0 ; y)}{\left(1-y^{2}\right)^{2}} \\
& +\frac{8\left(1+y^{2}\right)^{2} H(1,0,1,0 ; y)}{\left(16\left(1+y^{2}\right)^{2} H(1,1,0,0 ; y)\right.}
\end{aligned}
$$

$$
\Re c_{-2}=\frac{11}{12}+\frac{11\left(1+y^{2}\right) H(0 ; y)}{12\left(1-y^{2}\right)} ;
$$

$$
\begin{aligned}
\Re c_{-1}= & \frac{1}{36\left(1-y^{2}\right)^{2}}\left[-49+98 y^{2}-49 y^{4}+18 \zeta_{2}+144 y^{2} \zeta_{2}\right. \\
& \left.-162 y^{4} \zeta_{2}-18 \zeta_{3}-36 y^{2} \zeta_{3}-18 y^{4} \zeta_{3}\right] \\
& +\frac{\left(1+y^{2}\right)\left(-67+67 y^{2}+18 \zeta_{2}+162 y^{2} \zeta_{2}\right) H(0 ; y)}{36\left(1-y^{2}\right)^{2}} \\
& +\frac{\left(1+y^{2}\right) H(-1,0 ; y)}{1-y^{2}}-\frac{2 y^{2} H(0,0 ; y)}{1-y^{2}}-\frac{\left(1+y^{2}\right) H(1,0 ; y)}{1-y^{2}} \\
& +\frac{\left(1+y^{2}\right)^{2} H(0,-1,0 ; y)}{\left(1-y^{2}\right)^{2}}-\frac{2\left(y^{2}+y^{4}\right) H(0,0,0 ; y)}{\left(1-y^{2}\right)^{2}} \\
& -\frac{\left(1+y^{2}\right)^{2} H(0,1,0 ; y)}{\left(1-y^{2}\right)^{2}} ;
\end{aligned}
$$




$$
\begin{aligned}
& \Re c_{0}=-\frac{1}{540(1-y)^{3}(1+y)^{2}}\left[4345-4345 y-8690 y^{2}+8690 y^{3}\right. \\
& +4345 y^{4}-4345 y^{5}-8760 \zeta_{2}+10200 y \zeta_{2}-19440 \ln (2) y \zeta_{2} \\
& +25920 y^{2} \zeta_{2}-19440 \ln (2) y^{2} \zeta_{2}-13680 y^{3} \zeta_{2} \\
& +19440 \ln (2) y^{3} \zeta_{2}-17160 y^{4} \zeta_{2}+19440 \ln (2) y^{4} \zeta_{2} \\
& +3480 y^{5} \zeta_{2}+1701 \zeta_{2}^{2}-1701 y \zeta_{2}^{2}+10422 y^{2} \zeta_{2}{ }^{2} \\
& -19494 y^{3} \zeta_{2}^{2}+13257 y^{4} \zeta_{2}^{2}-13257 y^{5} \zeta_{2}^{2}-4410 \zeta_{3} \\
& -1530 y \zeta_{3}-2700 y^{2} \zeta_{3}+2700 y^{3} \zeta_{3}+7110 y^{4} \zeta_{3} \\
& \left.-1170 y^{5} \zeta_{3}\right] \\
& -\frac{1}{\left(1-y^{2}\right)^{2}}\left[37 \zeta_{2}-48 y^{2} \zeta_{2}+11 y^{4} \zeta_{2}-2 \zeta_{3}-4 y^{2} \zeta_{3}\right. \\
& \left.-2 y^{4} \zeta_{3}\right] H(-1 ; y) \\
& +\frac{1}{54(1-y)^{3}(1+y)^{2}}\left[-121-1133 y+1254 y^{2}+1254 y^{3}\right. \\
& -1133 y^{4}-121 y^{5}+198 \zeta_{2}+234 y \zeta_{2}-378 y^{2} \zeta_{2} \\
& -162 y^{3} \zeta_{2}+1908 y^{4} \zeta_{2}+1656 y^{5} \zeta_{2} \\
& +351 \zeta_{3}-351 y \zeta_{3}+594 y^{2} \zeta_{3}+702 y^{3} \zeta_{3}-405 y^{4} \zeta_{3} \\
& \left.+405 y^{5} \zeta_{3}\right] H(0 ; y) \\
& -\frac{2}{3\left(1-y^{2}\right)^{2}}\left[-52 \zeta_{2}+45 y \zeta_{2}+81 y^{2} \zeta_{2}+45 y^{3} \zeta_{2}\right. \\
& \left.+61 y^{4} \zeta_{2}+3 \zeta_{3}+6 y^{2} \zeta_{3}+3 y^{4} \zeta_{3}\right] H(1 ; y) \\
& +\frac{2\left(1+y^{2}\right)\left(-1+y^{2}-11 \zeta_{2}+y^{2} \zeta_{2}\right) H(-1,0 ; y)}{\left(1-y^{2}\right)^{2}} \\
& +\frac{\left(-19+19 y+46 y^{2}+98 y^{3}-7 y^{4}+7 y^{5}\right) \zeta_{2} H(0,-1 ; y)}{(1-y)^{3}(1+y)^{2}} \\
& -\frac{1}{18(1-y)^{3}(1+y)^{2}}\left[67+74 y-78 y^{2}-24 y^{3}+11 y^{4}-50 y^{5}\right. \\
& -18 \zeta_{2}+18 y \zeta_{2}-216 y^{2} \zeta_{2}+1152 y^{3} \zeta_{2}-666 y^{4} \zeta_{2} \\
& \left.+666 y^{5} \zeta_{2}\right] H(0,0 ; y) \\
& -\frac{\left(-11+11 y+14 y^{2}+106 y^{3}-35 y^{4}+35 y^{5}\right) \zeta_{2} H(0,1 ; y)}{(1-y)^{3}(1+y)^{2}} \\
& +\frac{1}{18\left(1-y^{2}\right)^{2}}\left[-53-102 y+102 y^{3}+53 y^{4}+252 \zeta_{2}+360 y^{2} \zeta_{2}\right. \\
& \left.+108 y^{4} \zeta_{2}\right] H(1,0 ; y)
\end{aligned}
$$




$$
\begin{aligned}
& -\frac{2\left(1+y^{2}\right) H(-1,-1,0 ; y)}{1-y^{2}}-\frac{4\left(-3+y^{2}\right) H(-1,0,0 ; y)}{1-y^{2}} \\
& +\frac{6\left(1+y^{2}\right) H(-1,1,0 ; y)}{1-y^{2}}-\frac{4 y\left(1-y+2 y^{2}\right) H(0,-1,0 ; y)}{(1-y)^{2}(1+y)} \\
& -\frac{\left(11+2 y-53 y^{2}+74 y^{3}+62 y^{4}\right) H(0,0,0 ; y)}{6(1-y)^{3}(1+y)} \\
& +\frac{2\left(-1+10 y-25 y^{2}+34 y^{3}\right) H(0,1,0 ; y)}{3(1-y)^{2}(1+y)} \\
& +\frac{6\left(1+y^{2}\right) H(1,-1,0 ; y)}{1-y^{2}} \\
& +\frac{\left(-61+175 y+35 y^{2}+79 y^{3}\right) H(1,0,0 ; y)}{6(1-y)^{2}(1+y)} \\
& -\frac{52\left(1+y^{2}\right) H(1,1,0 ; y)}{3\left(1-y^{2}\right)}-\frac{4\left(1+y^{2}\right)^{2} H(-1,0,-1,0 ; y)}{\left(1-y^{2}\right)^{2}} \\
& -\frac{2\left(-5-4 y^{2}+y^{4}\right) H(-1,0,0,0 ; y)}{\left(1-y^{2}\right)^{2}}+\frac{4\left(1+y^{2}\right)^{2} H(-1,0,1,0 ; y)}{\left(1-y^{2}\right)^{2}} \\
& -\frac{2\left(1+y^{2}\right)^{2} H(0,-1,-1,0 ; y)}{\left(1-y^{2}\right)^{2}} \\
& -\frac{2\left(-3+3 y+8 y^{2}+16 y^{3}-y^{4}+y^{5}\right) H(0,-1,0,0 ; y)}{(1-y)^{3}(1+y)^{2}} \\
& +\frac{6\left(1+y^{2}\right)^{2} H(0,-1,1,0 ; y)}{\left(1-y^{2}\right)^{2}}+\frac{2\left(1+8 y^{2}+7 y^{4}\right) H(0,0,-1,0 ; y)}{\left(1-y^{2}\right)^{2}} \\
& +\frac{3 y^{2}\left(-1+7 y-4 y^{2}+4 y^{3}\right) H(0,0,0,0 ; y)}{(1-y)^{3}(1+y)^{2}} \\
& +\frac{2\left(-1+y-22 y^{2}+2 y^{3}-11 y^{4}+11 y^{5}\right) H(0,0,1,0 ; y)}{(1-y)^{3}(1+y)^{2}} \\
& +\frac{6\left(1+y^{2}\right)^{2} H(0,1,-1,0 ; y)}{\left(1-y^{2}\right)^{2}} \\
& +\frac{2\left(-1+y-y^{2}+15 y^{3}-7 y^{4}+7 y^{5}\right) H(0,1,0,0 ; y)}{(1-y)^{3}(1+y)^{2}} \\
& -\frac{10\left(1+y^{2}\right)^{2} H(0,1,1,0 ; y)}{\left(1-y^{2}\right)^{2}}+\frac{4\left(1+y^{2}\right)^{2} H(1,0,-1,0 ; y)}{\left(1-y^{2}\right)^{2}} \\
& -\frac{2\left(3+4 y^{2}+y^{4}\right) H(1,0,0,0 ; y)}{\left(1-y^{2}\right)^{2}}-\frac{4\left(1+y^{2}\right)^{2} H(1,0,1,0 ; y)}{\left(1-y^{2}\right)^{2}} \text {; } \\
& \Re d_{-2}=-\frac{1}{3}-\frac{\left(1+y^{2}\right) H(0 ; y)}{3\left(1-y^{2}\right)} ;
\end{aligned}
$$




$$
\begin{aligned}
\Re d_{-1}= & \frac{5}{9}+\frac{5\left(1+y^{2}\right) H(0 ; y)}{9\left(1-y^{2}\right)} ; \\
\Re d_{0}= & -\frac{1}{27\left(1-y^{2}\right)}\left[-49+49 y^{2}+84 \zeta_{2}+288 y \zeta_{2}+156 y^{2} \zeta_{2}\right. \\
& \left.+36 \zeta_{3}+36 y^{2} \zeta_{3}\right] \\
& -\frac{4\left(-7-48 y-7 y^{2}+9 \zeta_{2}+9 y^{2} \zeta_{2}\right) H(0 ; y)}{27\left(1-y^{2}\right)} \\
& -\frac{16\left(1+y^{2}\right) \zeta_{2} H(1 ; y)}{3\left(1-y^{2}\right)}+\frac{2\left(5+12 y+5 y^{2}\right) H(0,0 ; y)}{9\left(1-y^{2}\right)} \\
& +\frac{4\left(5+12 y+5 y^{2}\right) H(1,0 ; y)}{9\left(1-y^{2}\right)}+\frac{2\left(1+y^{2}\right) H(0,0,0 ; y)}{3\left(1-y^{2}\right)} \\
& +\frac{4\left(1+y^{2}\right) H(0,1,0 ; y)}{3\left(1-y^{2}\right)}+\frac{4\left(1+y^{2}\right) H(1,0,0 ; y)}{3\left(1-y^{2}\right)} \\
& +\frac{8\left(1+y^{2}\right) H(1,1,0 ; y)}{3\left(1-y^{2}\right)} ;
\end{aligned}
$$

$\Re e_{-2}=0$;

$\Re e_{-1}=0$;

$$
\begin{aligned}
& \Re e_{0}=-\frac{1}{135\left(1-y^{2}\right)^{3}}\left[-2035-600 y+4905 y^{2}-4905 y^{4}+600 y^{5}\right. \\
& +2035 y^{6}+1260 \zeta_{2}-1080 y \zeta_{2}-19980 y^{2} \zeta_{2}+18360 y^{3} \zeta_{2} \\
& +19980 y^{4} \zeta_{2}-17280 y^{5} \zeta_{2}-1260 y^{6} \zeta_{2}+783 y \zeta_{2}{ }^{2} \\
& -3132 y^{2} \zeta_{2}{ }^{2}+32346 y^{3} \zeta_{2}{ }^{2}-3132 y^{4}{\zeta_{2}}^{2}+783 y^{5} \zeta_{2}{ }^{2} \\
& \left.+8640 y^{2} \zeta_{3}-8640 y^{4} \zeta_{3}\right] \\
& +\frac{384 y^{2} \zeta_{2} H(-1 ; y)}{\left(1-y^{2}\right)^{2}} \\
& -\frac{4}{27\left(1-y^{2}\right)^{3}}\left[-14+72 y-106 y^{2}-384 y^{3}-106 y^{4}+72 y^{5}\right. \\
& -14 y^{6}+9 \zeta_{2}-657 y^{2} \zeta_{2}+639 y^{4} \zeta_{2}+9 y^{6} \zeta_{2}-81 y \zeta_{3} \\
& \left.+324 y^{2} \zeta_{3}+378 y^{3} \zeta_{3}+324 y^{4} \zeta_{3}-81 y^{5} \zeta_{3}\right] H(0 ; y) \\
& -\frac{16 y\left(-1+2 y-y^{2}+\zeta_{2}-6 y \zeta_{2}+y^{2} \zeta_{2}\right) H(-1,0 ; y)}{(1-y)^{3}(1+y)} \\
& -\frac{8 y}{\left(1-y^{2}\right)^{3}}\left[-1+2 y^{2}-y^{4}+3 \zeta_{2}-12 y \zeta_{2}-78 y^{2} \zeta_{2}\right. \\
& \left.-12 y^{3} \zeta_{2}+3 y^{4} \zeta_{2}\right] H(0,-1 ; y) \\
& +\frac{2}{9(1-y)^{4}(1+y)^{3}}\left[5-23 y-101 y^{2}+531 y^{3}+27 y^{4}-353 y^{5}\right. \\
& +229 y^{6}+5 y^{7}-9 y \zeta_{2}+45 y^{2} \zeta_{2}+198 y^{3} \zeta_{2}-198 y^{4} \zeta_{2}
\end{aligned}
$$




$$
\begin{aligned}
& \left.-45 y^{5} \zeta_{2}+9 y^{6} \zeta_{2}\right] H(0,0 ; y) \\
& -\frac{32 y H(1,0 ; y)}{1-y^{2}}-\frac{128 y^{2} H(-1,0,0 ; y)}{\left(1-y^{2}\right)^{2}}+\frac{64 y^{2} H(0,-1,0 ; y)}{\left(1-y^{2}\right)^{2}} \\
& -\frac{2\left(-1+48 y^{2}+y^{4}\right) H(0,0,0 ; y)}{3\left(1-y^{2}\right)^{2}}-\frac{128 y^{2} H(0,1,0 ; y)}{\left(1-y^{2}\right)^{2}} \\
& +\frac{8 y\left(1-6 y+y^{2}\right) H(-1,0,0,0 ; y)}{(1-y)^{3}(1+y)} \\
& +\frac{8 y\left(1-4 y-26 y^{2}-4 y^{3}+y^{4}\right) H(0,-1,0,0 ; y)}{\left(1-y^{2}\right)^{3}} \\
& -\frac{8 y\left(1-6 y+y^{2}\right) H(0,0,-1,0 ; y)}{(1-y)^{3}(1+y)}+\frac{12(-1+y) y H(0,0,0,-1 ; y)}{(1+y)^{3}} \\
& -\frac{2(-1+y) y H(0,0,0,0 ; y)}{(1+y)^{3}}-\frac{256 y^{3} H(0,0,1,0 ; y)}{\left(1-y^{2}\right)^{3}}
\end{aligned}
$$

$$
\begin{aligned}
\Im b_{-2}= & \frac{1+y^{2}}{1-y^{2}}+\frac{\left(1+y^{2}\right)^{2} H(0 ; y)}{\left(1-y^{2}\right)^{2}} ; \\
\Im b_{-1}= & \frac{1+4 y-4 y^{3}-y^{4}-4 \zeta_{2}-8 y^{2} \zeta_{2}-4 y^{4} \zeta_{2}}{\left(1-y^{2}\right)^{2}} \\
& -\frac{\left(-1-8 y-8 y^{3}+y^{4}\right) H(0 ; y)}{\left(1-y^{2}\right)^{2}}+\frac{2\left(1+y^{2}\right) H(1 ; y)}{1-y^{2}} \\
& +\frac{3\left(1+y^{2}\right)^{2} H(0,0 ; y)}{\left(1-y^{2}\right)^{2}}+\frac{2\left(1+y^{2}\right)^{2} H(0,1 ; y)}{\left(1-y^{2}\right)^{2}} \\
& +\frac{4\left(1+y^{2}\right)^{2} H(1,0 ; y)}{\left(1-y^{2}\right)^{2}} ;
\end{aligned}
$$

$$
\begin{aligned}
\Im b_{0}= & -\frac{1}{(1-y)^{3}(1+y)^{2}}\left[-3-7 y+10 y^{2}+10 y^{3}-7 y^{4}-3 y^{5}-\zeta_{2}\right. \\
& +41 y \zeta_{2}-18 y^{2} \zeta_{2}+18 y^{3} \zeta_{2}-45 y^{4} \zeta_{2}+5 y^{5} \zeta_{2}+8 \zeta_{3} \\
& \left.-24 y \zeta_{3}-56 y^{2} \zeta_{3}-56 y^{3} \zeta_{3}-24 y^{4} \zeta_{3}+8 y^{5} \zeta_{3}\right] \\
& +\frac{4\left(2+4 y-4 y^{3}-2 y^{4}+\zeta_{2}+2 y^{2} \zeta_{2}+y^{4} \zeta_{2}\right) H(-1 ; y)}{\left(1-y^{2}\right)^{2}} \\
& +\frac{1}{(1-y)^{3}(1+y)^{2}}\left[3+12 y+36 y^{2}+22 y^{3}-47 y^{4}-26 y^{5}\right. \\
& -10 \zeta_{2}+6 y \zeta_{2}-28 y^{2} \zeta_{2}+20 y^{3} \zeta_{2}-18 y^{4} \zeta_{2} \\
& \left.+14 y^{5} \zeta_{2}\right] H(0 ; y) \\
& -\frac{\left(15+42 y-42 y^{3}-15 y^{4}+16 \zeta_{2}+32 y^{2} \zeta_{2}+16 y^{4} \zeta_{2}\right) H(1 ; y)}{\left(1-y^{2}\right)^{2}}
\end{aligned}
$$




$$
\begin{aligned}
& -\frac{2\left(5+16 y+6 y^{2}+16 y^{3}+5 y^{4}\right) H(-1,0 ; y)}{\left(1-y^{2}\right)^{2}} \\
& +\frac{4\left(1+6 y+2 y^{2}+6 y^{3}+y^{4}\right) H(0,-1 ; y)}{\left(1-y^{2}\right)^{2}} \\
& +\frac{\left(1+31 y-65 y^{2}-9 y^{3}+10 y^{5}\right) H(0,0 ; y)}{(1-y)^{3}(1+y)^{2}} \\
& -\frac{2\left(3+22 y+20 y^{2}+22 y^{3}+5 y^{4}\right) H(0,1 ; y)}{\left(1-y^{2}\right)^{2}} \\
& -\frac{\left(5-58 y-2 y^{2}-58 y^{3}+9 y^{4}\right) H(1,0 ; y)}{\left(1-y^{2}\right)^{2}} \\
& +\frac{4\left(1+y^{2}\right) H(1,1 ; y)}{1-y^{2}}+\frac{8\left(1+y^{2}\right)^{2} H(-1,0,-1 ; y)}{\left(1-y^{2}\right)^{2}} \\
& +\frac{8\left(-1+3 y+8 y^{2}+12 y^{3}+y^{4}+y^{5}\right) H(-1,0,0 ; y)}{(1-y)^{3}(1+y)^{2}} \\
& -\frac{8\left(1+y^{2}\right)^{2} H(-1,0,1 ; y)}{\left(1-y^{2}\right)^{2}}-\frac{4(1+y) H(0,-1,0 ; y)}{1-y} \\
& -\frac{4\left(-1+2 y^{2}+3 y^{4}\right) H(0,0,-1 ; y)}{\left(1-y^{2}\right)^{2}} \\
& -\frac{\left(-7+7 y-16 y^{2}+52 y^{3}-27 y^{4}+27 y^{5}\right) H(0,0,0 ; y)}{(1-y)^{3}(1+y)^{2}} \\
& +\frac{2\left(1+3 y-10 y^{2}+10 y^{3}-7 y^{4}+11 y^{5}\right) H(0,0,1 ; y)}{(1-y)^{3}(1+y)^{2}} \\
& +\frac{2\left(-3-5 y-36 y^{2}-16 y^{3}-15 y^{4}+7 y^{5}\right) H(0,1,0 ; y)}{(1-y)^{3}(1+y)^{2}} \\
& +\frac{4\left(1+y^{2}\right)^{2} H(0,1,1 ; y)}{\left(1-y^{2}\right)^{2}}+\frac{12\left(1+y^{2}\right)^{2} H(1,0,0 ; y)}{\left(1-y^{2}\right)^{2} H(1,0,1 ; y)} \\
& \left.+y^{2}\right)^{2}+\frac{16\left(1+y^{2}\right)^{2} H(1,1,0 ; y)}{\left(1-y^{2}\right)^{2}}
\end{aligned}
$$

$$
\begin{aligned}
\Im c_{-2}= & \frac{11\left(1+y^{2}\right)}{12\left(1-y^{2}\right)} ; \\
\Im c_{-1}= & \frac{\left(1+y^{2}\right)\left(-67+67 y^{2}+18 \zeta_{2}+18 y^{2} \zeta_{2}\right)}{36\left(1-y^{2}\right)^{2}} \\
& +\frac{\left(1+y^{2}\right) H(-1 ; y)}{1-y^{2}}-\frac{2 y^{2} H(0 ; y)}{1-y^{2}}-\frac{\left(1+y^{2}\right) H(1 ; y)}{1-y^{2}} \\
& +\frac{\left(1+y^{2}\right)^{2} H(0,-1 ; y)}{\left(1-y^{2}\right)^{2}}-\frac{2 y^{2}\left(1+y^{2}\right) H(0,0 ; y)}{\left(1-y^{2}\right)^{2}}
\end{aligned}
$$




$$
-\frac{\left(1+y^{2}\right)^{2} H(0,1 ; y)}{\left(1-y^{2}\right)^{2}} ;
$$

$$
\begin{aligned}
& \Im c_{0}=-\frac{1}{54(1-y)^{3}(1+y)^{2}}\left[121+1133 y-1254 y^{2}-1254 y^{3}+1133 y^{4}\right. \\
& +121 y^{5}-540 y^{2} \zeta_{2}+540 y^{3} \zeta_{2}+540 y^{4} \zeta_{2}-540 y^{5} \zeta_{2} \\
& -351 \zeta_{3}+351 y \zeta_{3}-594 y^{2} \zeta_{3}-702 y^{3} \zeta_{3}+405 y^{4} \zeta_{3} \\
& \left.-405 y^{5} \zeta_{3}\right] \\
& -\frac{2\left(1+y^{2}\right)\left(1-y^{2}+\zeta_{2}+y^{2} \zeta_{2}\right) H(-1 ; y)}{\left(1-y^{2}\right)^{2}} \\
& -\frac{1}{18(1-y)^{3}(1+y)^{2}}\left[67+74 y-78 y^{2}-24 y^{3}+11 y^{4}-50 y^{5}\right. \\
& -18 \zeta_{2}+18 y \zeta_{2}-108 y^{2} \zeta_{2}+396 y^{3} \zeta_{2}-234 y^{4} \zeta_{2} \\
& \left.+234 y^{5} \zeta_{2}\right] H(0 ; y) \\
& +\frac{1}{18\left(1-y^{2}\right)^{2}}\left[-53-102 y+102 y^{3}+53 y^{4}+36 \zeta_{2}\right. \\
& \left.+72 y^{2} \zeta_{2}+36 y^{4} \zeta_{2}\right] H(1 ; y) \\
& -\frac{2\left(1+y^{2}\right) H(-1,-1 ; y)}{1-y^{2}}-\frac{4\left(-3+y^{2}\right) H(-1,0 ; y)}{1-y^{2}} \\
& +\frac{6\left(1+y^{2}\right) H(-1,1 ; y)}{1-y^{2}}-\frac{4 y\left(1-y+2 y^{2}\right) H(0,-1 ; y)}{(1-y)^{2}(1+y)} \\
& -\frac{\left(11+2 y-53 y^{2}+74 y^{3}+62 y^{4}\right) H(0,0 ; y)}{6(1-y)^{3}(1+y)} \\
& +\frac{2\left(-1+10 y-25 y^{2}+34 y^{3}\right) H(0,1 ; y)}{3(1-y)^{2}(1+y)}+\frac{6\left(1+y^{2}\right) H(1,-1 ; y)}{1-y^{2}} \\
& +\frac{\left(-61+175 y+35 y^{2}+79 y^{3}\right) H(1,0 ; y)}{6(1-y)^{2}(1+y)}-\frac{52\left(1+y^{2}\right) H(1,1 ; y)}{3\left(1-y^{2}\right)} \\
& -\frac{4\left(1+y^{2}\right)^{2} H(-1,0,-1 ; y)}{\left(1-y^{2}\right)^{2}}-\frac{2\left(-5-4 y^{2}+y^{4}\right) H(-1,0,0 ; y)}{\left(1-y^{2}\right)^{2}} \\
& +\frac{4\left(1+y^{2}\right)^{2} H(-1,0,1 ; y)}{\left(1-y^{2}\right)^{2}}-\frac{2\left(1+y^{2}\right)^{2} H(0,-1,-1 ; y)}{\left(1-y^{2}\right)^{2}} \\
& -\frac{2\left(-3+3 y+8 y^{2}+16 y^{3}-y^{4}+y^{5}\right) H(0,-1,0 ; y)}{(1-y)^{3}(1+y)^{2}} \\
& +\frac{6\left(1+y^{2}\right)^{2} H(0,-1,1 ; y)}{\left(1-y^{2}\right)^{2}}+\frac{2\left(1+8 y^{2}+7 y^{4}\right) H(0,0,-1 ; y)}{\left(1-y^{2}\right)^{2}} \\
& +\frac{3 y^{2}\left(-1+7 y-4 y^{2}+4 y^{3}\right) H(0,0,0 ; y)}{(1-y)^{3}(1+y)^{2}}
\end{aligned}
$$




$$
\begin{aligned}
& +\frac{2\left(-1+y-22 y^{2}+2 y^{3}-11 y^{4}+11 y^{5}\right) H(0,0,1 ; y)}{(1-y)^{3}(1+y)^{2}} \\
& +\frac{6\left(1+y^{2}\right)^{2} H(0,1,-1 ; y)}{\left(1-y^{2}\right)^{2}} \\
& +\frac{2\left(-1+y-y^{2}+15 y^{3}-7 y^{4}+7 y^{5}\right) H(0,1,0 ; y)}{(1-y)^{3}(1+y)^{2}} \\
& -\frac{10\left(1+y^{2}\right)^{2} H(0,1,1 ; y)}{\left(1-y^{2}\right)^{2}}+\frac{4\left(1+y^{2}\right)^{2} H(1,0,-1 ; y)}{\left(1-y^{2}\right)^{2}} \\
& -\frac{2\left(3+4 y^{2}+y^{4}\right) H(1,0,0 ; y)}{\left(1-y^{2}\right)^{2}}-\frac{4\left(1+y^{2}\right)^{2} H(1,0,1 ; y)}{\left(1-y^{2}\right)^{2}}
\end{aligned}
$$

$\Im d_{-2}=-\frac{1+y^{2}}{3\left(1-y^{2}\right)} ;$

$$
\Im d_{-1}=\frac{5\left(1+y^{2}\right)}{9\left(1-y^{2}\right)} ;
$$

$$
\begin{aligned}
\Im d_{0}= & \frac{4\left(7+48 y+7 y^{2}\right)}{27\left(1-y^{2}\right)}+\frac{2\left(5+12 y+5 y^{2}\right) H(0 ; y)}{9\left(1-y^{2}\right)} \\
& +\frac{4\left(5+12 y+5 y^{2}\right) H(1 ; y)}{9\left(1-y^{2}\right)}+\frac{2\left(1+y^{2}\right) H(0,0 ; y)}{3\left(1-y^{2}\right)} \\
& +\frac{4\left(1+y^{2}\right) H(0,1 ; y)}{3\left(1-y^{2}\right)}+\frac{4\left(1+y^{2}\right) H(1,0 ; y)}{3\left(1-y^{2}\right)} \\
& +\frac{8\left(1+y^{2}\right) H(1,1 ; y)}{3\left(1-y^{2}\right)}
\end{aligned}
$$

$$
\begin{aligned}
\Im e_{-2}= & 0 ; \\
\Im e_{-1}= & 0 ; \\
\Im e_{0}= & \frac{4}{27\left(1-y^{2}\right)^{3}}\left[14-72 y+106 y^{2}+384 y^{3}+106 y^{4}-72 y^{5}+14 y^{6}\right. \\
& +216 y^{2} \zeta_{2}-216 y^{4} \zeta_{2}+81 y \zeta_{3}-324 y^{2} \zeta_{3}-378 y^{3} \zeta_{3} \\
& \left.-324 y^{4} \zeta_{3}+81 y^{5} \zeta_{3}\right] \\
& +\frac{16 y H(-1 ; y)}{1-y^{2}} \\
& +\frac{2}{9(1-y)^{4}(1+y)^{3}}\left[5-23 y-101 y^{2}+531 y^{3}+27 y^{4}-353 y^{5}\right. \\
& +229 y^{6}+5 y^{7}+9 y \zeta_{2}-45 y^{2} \zeta_{2}+378 y^{3} \zeta_{2}-378 y^{4} \zeta_{2} \\
& \left.+45 y^{5} \zeta_{2}-9 y^{6} \zeta_{2}\right] H(0 ; y) \\
& -\frac{32 y H(1 ; y)}{1-y^{2}}-\frac{128 y^{2} H(-1,0 ; y)}{\left(1-y^{2}\right)^{2}}+\frac{64 y^{2} H(0,-1 ; y)}{\left(1-y^{2}\right)^{2}}
\end{aligned}
$$




$$
\begin{aligned}
& -\frac{2\left(-1+48 y^{2}+y^{4}\right) H(0,0 ; y)}{3\left(1-y^{2}\right)^{2}}-\frac{128 y^{2} H(0,1 ; y)}{\left(1-y^{2}\right)^{2}} \\
& +\frac{8 y\left(1-6 y+y^{2}\right) H(-1,0,0 ; y)}{(1-y)^{3}(1+y)} \\
& +\frac{8 y\left(1-4 y-26 y^{2}-4 y^{3}+y^{4}\right) H(0,-1,0 ; y)}{\left(1-y^{2}\right)^{3}} \\
& -\frac{8 y\left(1-6 y+y^{2}\right) H(0,0,-1 ; y)}{(1-y)^{3}(1+y)} \\
& -\frac{2(-1+y) y H(0,0,0 ; y)}{(1+y)^{3}}-\frac{256 y^{3} H(0,0,1 ; y)}{\left(1-y^{2}\right)^{3}}
\end{aligned}
$$

\subsubsection{The Pseudoscalar Case}

$\Re \bar{b}_{-2}=\Re b_{-2} ;$

$$
\begin{aligned}
& \Re \bar{b}_{-1}= \frac{\left(1+y^{2}\right) H(0,0 ; y)}{1-y^{2}}+\frac{2\left(1+y^{2}\right) H(1,0 ; y)}{1-y^{2}} \\
&+\frac{3\left(1+y^{2}\right)^{2} H(0,0,0 ; y)}{\left(1-y^{2}\right)^{2}}+\frac{2\left(1+y^{2}\right)^{2} H(0,1,0 ; y)}{\left(1-y^{2}\right)^{2}} \\
&+\frac{4\left(1+y^{2}\right)^{2} H(1,0,0 ; y)}{\left(1-y^{2}\right)^{2}}-\frac{12\left(1+y^{2}\right)^{2} H(1 ; y) \zeta_{2}}{\left(1-y^{2}\right)^{2}} \\
&-\frac{\left(1+y^{2}\right) H(0 ; y)\left(-1+y^{2}+10 \zeta_{2}+10 y^{2} \zeta_{2}\right)}{\left(1-y^{2}\right)^{2}} \\
&-\frac{-1+y^{2}+4 \zeta_{2}+4 y^{2} \zeta_{2}}{1-y^{2}} ; \\
& \Re \bar{b}_{0}=-2\left(5+12 y-18 y^{2}+12 y^{3}+5 y^{4}\right) H(-1,0,0 ; y) \\
&\left(1-y^{2}\right)^{2} \\
&+\frac{4\left(1+y^{2}\right) H(0,-1,0 ; y)}{(1-y)^{2}} \\
&-\frac{\left(-1-9 y+9 y^{2}+71 y^{3}+16 y^{4}+10 y^{5}\right) H(0,0,0 ; y)}{(1-y)^{2}(1+y)^{3}} \\
&-\frac{2\left(3+14 y-4 y^{2}+14 y^{3}+5 y^{4}\right) H(0,1,0 ; y)}{\left(1-y^{2}\right)^{2}} \\
&-\frac{\left(5-14 y+46 y^{2}-14 y^{3}+9 y^{4}\right) H(1,0,0 ; y)}{\left(1-y^{2}\right)^{2}} \\
&+\frac{4\left(1+y^{2}\right) H(1,1,0 ; y)}{1-y^{2}} \\
&+\frac{8\left(1+y^{2}\right)^{2} H(-1,0,-1,0 ; y)}{\left(1-y^{2}\right)^{2}}
\end{aligned}
$$




$$
\begin{aligned}
& -\frac{8\left(1-2 y+2 y^{2}+2 y^{3}+y^{4}\right) H(-1,0,0,0 ; y)}{\left(1-y^{2}\right)^{2}} \\
& -\frac{8\left(1+y^{2}\right)^{2} H(-1,0,1,0 ; y)}{\left(1-y^{2}\right)^{2}} \\
& -\frac{4\left(1+4 y+2 y^{2}+4 y^{3}+y^{4}\right) H(0,-1,0,0 ; y)}{(1-y)(1+y)^{3}} \\
& -\frac{4\left(-1+2 y^{2}+3 y^{4}\right) H(0,0,-1,0 ; y)}{\left(1-y^{2}\right)^{2}} \\
& +\frac{\left(7+7 y+24 y^{2}+44 y^{3}+27 y^{4}+27 y^{5}\right) H(0,0,0,0 ; y)}{(1-y)^{2}(1+y)^{3}} \\
& +\frac{2\left(-1-5 y+10 y^{2}+10 y^{3}+15 y^{4}+11 y^{5}\right) H(0,0,1,0 ; y)}{(1-y)^{2}(1+y)^{3}} \\
& +\frac{2\left(3+11 y+12 y^{2}+8 y^{3}-y^{4}+7 y^{5}\right) H(0,1,0,0 ; y)}{(1-y)^{2}(1+y)^{3}} \\
& +\frac{4\left(1+y^{2}\right)^{2} H(0,1,1,0 ; y)}{\left(1-y^{2}\right)^{2}} \\
& +\frac{12\left(1+y^{2}\right)^{2} H(1,0,0,0 ; y)}{\left(1-y^{2}\right)^{2}} \\
& +\frac{8\left(1+y^{2}\right)^{2} H(1,0,1,0 ; y)}{\left(1-y^{2}\right)^{2}} \\
& +\frac{H(1-y)^{2}(1+y)^{3}}{\left(1-3-14 y+6 y^{2}+8 y^{3}-35 y^{4}-26 y^{5}+24 \zeta_{2}\right.} \\
& +\frac{48\left(1+y^{2}\right)^{2} H(1,1 ; y) \zeta_{2}}{\left(1-y^{2}\right)^{2}} \\
& +\frac{16\left(1+y^{2}\right)^{2} H(1,1,0,0 ; y)}{\left(1-y^{2}\right)^{2}} \\
& +\frac{2\left(-5+25 y-17 y^{2}+13 y^{3}\right) H(1 ; y) \zeta_{2}}{(1-y)(1+y)^{2}} \\
& +\frac{12\left(1+4 y+2 y^{2}+4 y^{3}+y^{4}\right) H(0,-1 ; y) \zeta_{2}}{(1-y)(1+y)^{3}}\left[2-2 y^{4}+5 \zeta_{2}-8 y \zeta_{2}+10 y^{2} \zeta_{2}\right. \\
& +2 y y^{2}(1+y)^{3}
\end{aligned}
$$




$$
\begin{aligned}
& \left.+28 y \zeta_{2}+76 y^{2} \zeta_{2}+108 y^{3} \zeta_{2}+64 y^{4} \zeta_{2}+68 y^{5} \zeta_{2}\right] \\
& +\frac{2 H(-1 ; y)}{\left(1-y^{2}\right)^{2}}\left[15 \zeta_{2}+36 y \zeta_{2}-54 y^{2} \zeta_{2}+36 y^{3} \zeta_{2}+15 y^{4} \zeta_{2}\right. \\
& \left.-2 \zeta_{3}-4 y^{2} \zeta_{3}-2 y^{4} \zeta_{3}\right] \\
& +\frac{1}{20(1-y)^{2}(1+y)^{3}}\left[145+145 y-290 y^{2}-290 y^{3}+145 y^{4}+145 y^{5}\right. \\
& -160 \zeta_{2}-600 y \zeta_{2}+1960 y^{2} \zeta_{2}+2120 y^{3} \zeta_{2}-1800 y^{4} \zeta_{2} \\
& -1520 y^{5} \zeta_{2}-480 y \ln (2) \zeta_{2}-1440 y^{2} \ln (2) \zeta_{2}-1440 y^{3} \ln (2) \zeta_{2} \\
& -480 y^{4} \ln (2) \zeta_{2}+272 \zeta_{2}{ }^{2}+376 y \zeta_{2}{ }^{2}+912 y^{2} \zeta_{2}{ }^{2}+416 y^{3} \zeta_{2}{ }^{2} \\
& +288 y^{4} \zeta_{2}^{2}+392 y^{5} \zeta_{2}^{2}-280 \zeta_{3}-280 y^{2} \zeta_{3}-280 y^{3} \zeta_{3} \\
& \left.+80 y^{4} \zeta_{3}-200 y^{5} \zeta_{3}\right] \\
& +\frac{H(0 ; y)}{(1-y)^{2}(1+y)^{3}}\left[3+y-2 y^{2}+2 y^{3}-y^{4}-3 y^{5}-\zeta_{2}-21 y \zeta_{2}\right. \\
& +16 y^{2} \zeta_{2}+140 y^{3} \zeta_{2}+33 y^{4} \zeta_{2}+25 y^{5} \zeta_{2}-8 \zeta_{3}+8 y \zeta_{3} \\
& \left.+8 y^{2} \zeta_{3}-8 y^{3} \zeta_{3}-8 y^{4} \zeta_{3}+8 y^{5} \zeta_{3}\right] ;
\end{aligned}
$$

$\Re \bar{c}_{-2}=\Re c_{-2} ;$

$$
\Re \bar{c}_{-1}=\Re c_{-1} ;
$$

$$
\begin{aligned}
\Re \bar{c}_{0}= & \frac{-2\left(1+y^{2}\right) H(-1,-1,0 ; y)}{1-y^{2}} \\
& +\frac{4\left(3+2 y-4 y^{2}+2 y^{3}+y^{4}\right) H(-1,0,0 ; y)}{\left(1-y^{2}\right)^{2}} \\
& +\frac{6\left(1+y^{2}\right) H(-1,1,0 ; y)}{1-y^{2}} \\
& -\frac{4 y\left(1-y+2 y^{2}\right) H(0,-1,0 ; y)}{(1-y)^{2}(1+y)} \\
& +\frac{\left(-11-35 y-45 y^{2}+189 y^{3}+128 y^{4}+62 y^{5}\right) H(0,0,0 ; y)}{6(1-y)^{2}(1+y)^{3}} \\
& +\frac{2\left(-1+15 y-15 y^{2}+15 y^{3}+34 y^{4}\right) H(0,1,0 ; y)}{3\left(1-y^{2}\right)^{2}} \\
& +\frac{6\left(1+y^{2}\right) H(1,-1,0 ; y)}{1-y^{2}} \\
& +\frac{\left(-61+6 y+66 y^{2}+6 y^{3}+79 y^{4}\right) H(1,0,0 ; y)}{6\left(1-y^{2}\right)^{2}} \\
& -\frac{52\left(1+y^{2}\right) H(1,1,0 ; y)}{3\left(1-y^{2}\right)}
\end{aligned}
$$




$$
\begin{aligned}
& -\frac{4\left(1+y^{2}\right)^{2} H(-1,0,-1,0 ; y)}{\left(1-y^{2}\right)^{2}} \\
& -\frac{2\left(-5-4 y^{2}+y^{4}\right) H(-1,0,0,0 ; y)}{\left(1-y^{2}\right)^{2}} \\
& +\frac{4\left(1+y^{2}\right)^{2} H(-1,0,1,0 ; y)}{\left(1-y^{2}\right)^{2}} \\
& -\frac{2\left(1+y^{2}\right)^{2} H(0,-1,-1,0 ; y)}{\left(1-y^{2}\right)^{2}} \\
& +\frac{2\left(3+3 y+8 y^{3}+y^{4}+y^{5}\right) H(0,-1,0,0 ; y)}{(1-y)^{2}(1+y)^{3}} \\
& +\frac{6\left(1+y^{2}\right)^{2} H(0,-1,1,0 ; y)}{\left(1-y^{2}\right)^{2}} \\
& +\frac{2\left(1+8 y^{2}+7 y^{4}\right) H(0,0,-1,0 ; y)}{\left(1-y^{2}\right)^{2}} \\
& -\frac{y^{2}\left(7+17 y+12 y^{2}+12 y^{3}\right) H(0,0,0,0 ; y)}{(1-y)^{2}(1+y)^{3}} \\
& -\frac{2\left(1+y+14 y^{2}+10 y^{3}+11 y^{4}+11 y^{5}\right) H(0,0,1,0 ; y)}{(1-y)^{2}(1+y)^{3}} \\
& +\frac{6\left(1+y^{2}\right)^{2} H(0,1,-1,0 ; y)}{\left(1-y^{2}\right)^{2}} \\
& -\frac{2\left(1+y+5 y^{2}+11 y^{3}+7 y^{4}+7 y^{5}\right) H(0,1,0,0 ; y)}{(1-y)^{2}(1+y)^{3}} \\
& -\frac{10\left(1+y^{2}\right)^{2} H(0,1,1,0 ; y)}{\left(1-y^{2}\right)^{2}} \\
& +\frac{4\left(1+y^{2}\right)^{2} H(1,0,-1,0 ; y)}{\left(1-y^{2}\right)^{2}} \\
& -\frac{2\left(3+4 y^{2}+y^{4}\right) H(1,0,0,0 ; y)}{\left(1-y^{2}\right)^{2}} \\
& -\frac{4\left(1+y^{2}\right)^{2} H(1,0,1,0 ; y)}{\left(1-y^{2}\right)^{2}} \\
& -\frac{\left(19+19 y+2 y^{2}+50 y^{3}+7 y^{4}+7 y^{5}\right) H(0,-1 ; y) \zeta_{2}}{(1-y)^{2}(1+y)^{3}} \\
& +\frac{\left(11+11 y+34 y^{2}+58 y^{3}+35 y^{4}+35 y^{5}\right) H(0,1 ; y) \zeta_{2}}{(1-y)^{2}(1+y)^{3}} \\
& +\frac{2\left(1+y^{2}\right) H(-1,0 ; y)\left(-1+y^{2}-11 \zeta_{2}+y^{2} \zeta_{2}\right)}{\left(1-y^{2}\right)^{2}}
\end{aligned}
$$




$$
\begin{aligned}
& +\frac{H(1,0 ; y)}{18\left(1-y^{2}\right)^{2}}\left[-53+54 y-54 y^{3}+53 y^{4}+252 \zeta_{2}+360 y^{2} \zeta_{2}\right. \\
& \left.+108 y^{4} \zeta_{2}\right] \\
& +\frac{H(0,0 ; y)}{18(1-y)^{2}(1+y)^{3}}\left[-67-148 y-36 y^{2}-90 y^{3}-185 y^{4}-50 y^{5}+18 \zeta_{2}\right. \\
& \left.+18 y \zeta_{2}+504 y^{2} \zeta_{2}+864 y^{3} \zeta_{2}+666 y^{4} \zeta_{2}+666 y^{5} \zeta_{2}\right] \\
& -\frac{2\left(1+y^{2}\right) H(1 ; y)\left(-52 \zeta_{2}-9 y \zeta_{2}+61 y^{2} \zeta_{2}+3 \zeta_{3}+3 y^{2} \zeta_{3}\right)}{3\left(1-y^{2}\right)^{2}} \\
& -\frac{H(-1 ; y)}{\left(1-y^{2}\right)^{2}}\left[37 \zeta_{2}+24 y \zeta_{2}-48 y^{2} \zeta_{2}+24 y^{3} \zeta_{2}+11 y^{4} \zeta_{2}\right. \\
& \left.-2 \zeta_{3}-4 y^{2} \zeta_{3}-2 y^{4} \zeta_{3}\right] \\
& +\frac{1}{540(1-y)^{2}(1+y)^{3}}\left[-4345-4345 y+8690 y^{2}+8690 y^{3}-4345 y^{4}\right. \\
& -4345 y^{5}+8760 \zeta_{2}+12000 y \zeta_{2}-21960 y^{2} \zeta_{2}-15480 y^{3} \zeta_{2} \\
& +13200 y^{4} \zeta_{2}+3480 y^{5} \zeta_{2}+6480 y \ln (2) \zeta_{2}+19440 y^{2} \ln (2) \zeta_{2} \\
& +19440 y^{3} \ln (2) \zeta_{2}+6480 y^{4} \ln (2) \zeta_{2}-1701 \zeta_{2}^{2}-1701 y \zeta_{2}^{2} \\
& -16902 y^{2}{\zeta_{2}}^{2}-13014 y^{3} \zeta_{2}{ }^{2}-13257 y^{4} \zeta_{2}{ }^{2}-13257 y^{5} \zeta_{2}{ }^{2} \\
& +4410 \zeta_{3}+4950 y \zeta_{3}+2700 y^{2} \zeta_{3}+2700 y^{3} \zeta_{3}-630 y^{4} \zeta_{3} \\
& \left.-1170 y^{5} \zeta_{3}\right] \\
& -\frac{H(0 ; y)}{54(1-y)^{2}(1+y)^{3}}\left[121+283 y+162 y^{2}-162 y^{3}-283 y^{4}-121 y^{5}\right. \\
& -198 \zeta_{2}-630 y \zeta_{2}-1350 y^{2} \zeta_{2}+2862 y^{3} \zeta_{2}+2844 y^{4} \zeta_{2} \\
& +1656 y^{5} \zeta_{2}-351 \zeta_{3}-351 y \zeta_{3}-162 y^{2} \zeta_{3}+270 y^{3} \zeta_{3} \\
& \left.+405 y^{4} \zeta_{3}+405 y^{5} \zeta_{3}\right]
\end{aligned}
$$

$\Re \bar{d}_{-2}=\Re d_{-2} ;$

$\Re \bar{d}_{-1}=\Re d_{-1} ;$

$$
\begin{aligned}
\Re \bar{d}_{0}= & \frac{10\left(1+y^{2}\right) H(0,0 ; y)}{9\left(1-y^{2}\right)}+\frac{20\left(1+y^{2}\right) H(1,0 ; y)}{9\left(1-y^{2}\right)} \\
& +\frac{2\left(1+y^{2}\right) H(0,0,0 ; y)}{3\left(1-y^{2}\right)}+\frac{4\left(1+y^{2}\right) H(0,1,0 ; y)}{3\left(1-y^{2}\right)} \\
& +\frac{4\left(1+y^{2}\right) H(1,0,0 ; y)}{3\left(1-y^{2}\right)}+\frac{8\left(1+y^{2}\right) H(1,1,0 ; y)}{3\left(1-y^{2}\right)} \\
& -\frac{16\left(1+y^{2}\right) H(1 ; y) \zeta_{2}}{3\left(1-y^{2}\right)}-\frac{4\left(1+y^{2}\right) H(0 ; y)\left(-7+9 \zeta_{2}\right)}{27\left(1-y^{2}\right)}
\end{aligned}
$$




$$
-\frac{-49+49 y^{2}+84 \zeta_{2}+156 y^{2} \zeta_{2}+36 \zeta_{3}+36 y^{2} \zeta_{3}}{27\left(1-y^{2}\right)}
$$

$$
\begin{aligned}
\Re \bar{e}_{-2}= & \Re e_{-2}=0 ; \\
\Re \bar{e}_{-1}= & \Re e_{-1}=0 ; \\
\Re \bar{e}_{0}= & \frac{2\left(1+2 y+26 y^{2}+2 y^{3}+y^{4}\right) H(0,0,0 ; y)}{3(1-y)(1+y)^{3}}+\frac{8 y H(-1,0,0,0 ; y)}{1-y^{2}} \\
& +\frac{8 y H(0,-1,0,0 ; y)}{1-y^{2}}-\frac{8 y H(0,0,-1,0 ; y)}{1-y^{2}}-\frac{12 y H(0,0,0,-1 ; y)}{1-y^{2}} \\
& +\frac{2 y H(0,0,0,0 ; y)}{1-y^{2}}-\frac{16 y H(-1,0 ; y) \zeta_{2}}{1-y^{2}}-\frac{24 y H(0,-1 ; y) \zeta_{2}}{1-y^{2}} \\
& +\frac{2 H(0,0 ; y)}{9(1-y)^{4}(1+y)^{2}}\left[5-22 y+59 y^{2}-116 y^{3}+59 y^{4}-22 y^{5}+5 y^{6}\right. \\
& \left.-9 y \zeta_{2}+18 y^{2} \zeta_{2}-18 y^{4} \zeta_{2}+9 y^{5} \zeta_{2}\right] \\
& -\frac{4 H(0 ; y)}{27\left(1-y^{2}\right)^{3}}\left[-14+12 y+38 y^{2}+24 y^{3}+38 y^{4}+12 y^{5}-14 y^{6}\right. \\
& +9 \zeta_{2}+207 y^{2} \zeta_{2}-432 y^{3} \zeta_{2}+207 y^{4} \zeta_{2}+9 y^{6} \zeta_{2}-81 y \zeta_{3} \\
& \left.+162 y^{3} \zeta_{3}-81 y^{5} \zeta_{3}\right] \\
& +\frac{1}{135\left(1-y^{2}\right)^{2}}\left[2035-120 y-4310 y^{2}-120 y^{3}+2035 y^{4}-1260 \zeta_{2}\right. \\
& +540 y \zeta_{2}+1440 y^{2} \zeta_{2}+540 y^{3} \zeta_{2}-1260 y^{4} \zeta_{2}-783 y \zeta_{2}{ }^{2} \\
& \left.+783 y^{3} \zeta_{2}^{2}\right] ;
\end{aligned}
$$

$$
\begin{aligned}
\Im \bar{b}_{-2}= & \Im b_{-2} ; \\
\Im \bar{b}_{-1}= & \frac{\left(1+y^{2}\right) H(0 ; y)}{1-y^{2}}+\frac{2\left(1+y^{2}\right) H(1 ; y)}{1-y^{2}}+\frac{3\left(1+y^{2}\right)^{2} H(0,0 ; y)}{\left(1-y^{2}\right)^{2}} \\
& +\frac{2\left(1+y^{2}\right)^{2} H(0,1 ; y)}{\left(1-y^{2}\right)^{2}}+\frac{4\left(1+y^{2}\right)^{2} H(1,0 ; y)}{\left(1-y^{2}\right)^{2}} \\
& -\frac{\left(1+y^{2}\right)\left(-1+y^{2}+4 \zeta_{2}+4 y^{2} \zeta_{2}\right)}{\left(1-y^{2}\right)^{2}} ; \\
\Im \bar{b}_{0}= & \frac{-2\left(5+12 y-18 y^{2}+12 y^{3}+5 y^{4}\right) H(-1,0 ; y)}{\left(1-y^{2}\right)^{2}} \\
& +\frac{4\left(1+y^{2}\right) H(0,-1 ; y)}{(1-y)^{2}}
\end{aligned}
$$




$$
\begin{aligned}
& -\frac{\left(-1-9 y+9 y^{2}+71 y^{3}+16 y^{4}+10 y^{5}\right) H(0,0 ; y)}{(1-y)^{2}(1+y)^{3}} \\
& -\frac{2\left(3+14 y-4 y^{2}+14 y^{3}+5 y^{4}\right) H(0,1 ; y)}{\left(1-y^{2}\right)^{2}} \\
& -\frac{\left(5-14 y+46 y^{2}-14 y^{3}+9 y^{4}\right) H(1,0 ; y)}{\left(1-y^{2}\right)^{2}} \\
& +\frac{4\left(1+y^{2}\right) H(1,1 ; y)}{1-y^{2}} \\
& +\frac{8\left(1+y^{2}\right)^{2} H(-1,0,-1 ; y)}{\left(1-y^{2}\right)^{2}} \\
& -\frac{8\left(1-2 y+2 y^{2}+2 y^{3}+y^{4}\right) H(-1,0,0 ; y)}{\left(1-y^{2}\right)^{2}} \\
& -\frac{8\left(1+y^{2}\right)^{2} H(-1,0,1 ; y)}{\left(1-y^{2}\right)^{2}} \\
& -\frac{4\left(1+4 y+2 y^{2}+4 y^{3}+y^{4}\right) H(0,-1,0 ; y)}{(1-y)(1+y)^{3}} \\
& -\frac{4\left(-1+2 y^{2}+3 y^{4}\right) H(0,0,-1 ; y)}{\left(1-y^{2}\right)^{2}} \\
& +\frac{\left(7+7 y+24 y^{2}+44 y^{3}+27 y^{4}+27 y^{5}\right) H(0,0,0 ; y)}{(1-y)^{2}(1+y)^{3}} \\
& +\frac{2\left(-1-5 y+10 y^{2}+10 y^{3}+15 y^{4}+11 y^{5}\right) H(0,0,1 ; y)}{(1-y)^{2}(1+y)^{3}} \\
& +\frac{2\left(3+11 y+12 y^{2}+8 y^{3}-y^{4}+7 y^{5}\right) H(0,1,0 ; y)}{(1-y)^{2}(1+y)^{3}} \\
& +\frac{4\left(1+y^{2}\right)^{2} H(0,1,1 ; y)}{\left(1-y^{2}\right)^{2}} \\
& +\frac{12\left(1+y^{2}\right)^{2} H(1,0,0 ; y)}{\left(1-y^{2}\right)^{2}} \\
& +\frac{8\left(1+y^{2}\right)^{2} H(1,0,1 ; y)}{\left(1-y^{2}\right)^{2}} \\
& +\frac{16\left(1+y^{2}\right)^{2} H(1,1,0 ; y)}{\left(1-y^{2}\right)^{2}} \\
& +\frac{4\left(1+y^{2}\right) H(-1 ; y)\left(2-2 y^{2}+\zeta_{2}+y^{2} \zeta_{2}\right)}{\left(1-y^{2}\right)^{2}} \\
& -\frac{H(0 ; y)}{\left(1-y^{2}\right)^{2}}\left(-3-11 y+17 y^{2}-9 y^{3}-26 y^{4}+10 \zeta_{2}+4 y \zeta_{2}\right. \\
& \left.+24 y^{2} \zeta_{2}-4 y^{3} \zeta_{2}+14 y^{4} \zeta_{2}\right]
\end{aligned}
$$




$$
\begin{aligned}
& -\frac{H(1 ; y)\left(15-2 y+2 y^{3}-15 y^{4}+16 \zeta_{2}+32 y^{2} \zeta_{2}+16 y^{4} \zeta_{2}\right)}{\left(1-y^{2}\right)^{2}} \\
& -\frac{1}{(1-y)(1+y)^{3}}\left[-3-4 y-2 y^{2}-4 y^{3}-3 y^{4}-\zeta_{2}+2 y \zeta_{2}\right. \\
& \left.+4 y^{2} \zeta_{2}+6 y^{3} \zeta_{2}+5 y^{4} \zeta_{2}+8 \zeta_{3}-8 y^{2} \zeta_{3}+8 y^{4} \zeta_{3}\right]
\end{aligned}
$$

$\Im \bar{c}_{-2}=\Im c_{-2} ;$

$$
\Im \bar{c}_{-1}=\Im c_{-1} ;
$$

$$
\begin{aligned}
\Im \bar{c}_{0}= & \frac{-2\left(1+y^{2}\right) H(-1,-1 ; y)}{1-y^{2}} \\
& +\frac{4\left(3+2 y-4 y^{2}+2 y^{3}+y^{4}\right) H(-1,0 ; y)}{\left(1-y^{2}\right)^{2}} \\
& +\frac{6\left(1+y^{2}\right) H(-1,1 ; y)}{1-y^{2}} \\
& -\frac{4 y\left(1-y+2 y^{2}\right) H(0,-1 ; y)}{(1-y)^{2}(1+y)} \\
& +\frac{\left(-11-35 y-45 y^{2}+189 y^{3}+128 y^{4}+62 y^{5}\right) H(0,0 ; y)}{6(1-y)^{2}(1+y)^{3}} \\
& +\frac{2\left(-1+15 y-15 y^{2}+15 y^{3}+34 y^{4}\right) H(0,1 ; y)}{3\left(1-y^{2}\right)^{2}} \\
& +\frac{6\left(1+y^{2}\right) H(1,-1 ; y)}{1-y^{2}} \\
& +\frac{\left(-61+6 y+66 y^{2}+6 y^{3}+79 y^{4}\right) H(1,0 ; y)}{6\left(1-y^{2}\right)^{2}} \\
& -\frac{52\left(1+y^{2}\right) H(1,1 ; y)}{3\left(1-y^{2}\right)} \\
& -\frac{4\left(1+y^{2}\right)^{2} H(-1,0,-1 ; y)}{\left(1-y^{2}\right)^{2}} \\
& -\frac{2\left(-5-4 y^{2}+y^{4}\right) H(-1,0,0 ; y)}{\left(1-y^{2}\right)^{2}} \\
& +\frac{4\left(1+y^{2}\right)^{2} H(-1,0,1 ; y)}{\left(1-y^{2}\right)^{2}} \\
& -\frac{2\left(1+y^{2}\right)^{2} H(0,-1,-1 ; y)}{\left(1-y^{2}\right)^{2}} \\
(1-y)^{2}(1+y)^{3} & \frac{2\left(3+3 y+8 y^{3}+y^{4}+y^{5}\right) H(0,-1,0 ; y)}{(1-y)} \\
& \\
&
\end{aligned}
$$




$$
\begin{aligned}
& +\frac{6\left(1+y^{2}\right)^{2} H(0,-1,1 ; y)}{\left(1-y^{2}\right)^{2}} \\
& +\frac{2\left(1+8 y^{2}+7 y^{4}\right) H(0,0,-1 ; y)}{\left(1-y^{2}\right)^{2}} \\
& -\frac{y^{2}\left(7+17 y+12 y^{2}+12 y^{3}\right) H(0,0,0 ; y)}{(1-y)^{2}(1+y)^{3}} \\
& -\frac{2\left(1+y+14 y^{2}+10 y^{3}+11 y^{4}+11 y^{5}\right) H(0,0,1 ; y)}{(1-y)^{2}(1+y)^{3}} \\
& +\frac{6\left(1+y^{2}\right)^{2} H(0,1,-1 ; y)}{\left(1-y^{2}\right)^{2}} \\
& -\frac{2\left(1+y+5 y^{2}+11 y^{3}+7 y^{4}+7 y^{5}\right) H(0,1,0 ; y)}{(1-y)^{2}(1+y)^{3}} \\
& -\frac{10\left(1+y^{2}\right)^{2} H(0,1,1 ; y)}{\left(1-y^{2}\right)^{2}} \\
& +\frac{4\left(1+y^{2}\right)^{2} H(1,0,-1 ; y)}{\left(1-y^{2}\right)^{2}} \\
& -\frac{2\left(3+4 y^{2}+y^{4}\right) H(1,0,0 ; y)}{\left(1-y^{2}\right)^{2}} \\
& -\frac{4\left(1+y^{2}\right)^{2} H(1,0,1 ; y)}{\left(1-y^{2}\right)^{2}} \\
& -\frac{2\left(1+y^{2}\right) H(-1 ; y)\left(1-y^{2}+\zeta_{2}+y^{2} \zeta_{2}\right)}{\left(1-y^{2}\right)^{2}} \\
& +\frac{H(1 ; y)}{18\left(1-y^{2}\right)^{2}}\left[-53+54 y-54 y^{3}+53 y^{4}+36 \zeta_{2}+72 y^{2} \zeta_{2}\right. \\
& \left.+36 y^{4} \zeta_{2}\right] \\
& +\frac{H(0 ; y)}{18\left(1-y^{2}\right)^{2}}\left[-67-81 y+45 y^{2}-135 y^{3}-50 y^{4}+18 \zeta_{2}\right. \\
& \left.+252 y^{2} \zeta_{2}+234 y^{4} \zeta_{2}\right] \\
& +\frac{1}{54(1-y)^{2}(1+y)^{3}}\left[-121-283 y-162 y^{2}+162 y^{3}+283 y^{4}\right. \\
& +121 y^{5}+540 y^{2} \zeta_{2}+540 y^{3} \zeta_{2}-540 y^{4} \zeta_{2}-540 y^{5} \zeta_{2} \\
& +351 \zeta_{3}+351 y \zeta_{3}+162 y^{2} \zeta_{3}-270 y^{3} \zeta_{3}-405 y^{4} \zeta_{3} \\
& \left.-405 y^{5} \zeta_{3}\right] \text {; }
\end{aligned}
$$

$\Im \bar{d}_{-2}=\Im d_{-2} ;$

$\Im \bar{d}_{-1}=\Im d_{-1} ;$ 


$$
\begin{aligned}
& \Im \bar{d}_{0}=\frac{28\left(1+y^{2}\right)}{27\left(1-y^{2}\right)}+\frac{10\left(1+y^{2}\right) H(0 ; y)}{9\left(1-y^{2}\right)}+\frac{20\left(1+y^{2}\right) H(1 ; y)}{9\left(1-y^{2}\right)} \\
& +\frac{2\left(1+y^{2}\right) H(0,0 ; y)}{3\left(1-y^{2}\right)}+\frac{4\left(1+y^{2}\right) H(0,1 ; y)}{3\left(1-y^{2}\right)} \\
& +\frac{4\left(1+y^{2}\right) H(1,0 ; y)}{3\left(1-y^{2}\right)}+\frac{8\left(1+y^{2}\right) H(1,1 ; y)}{3\left(1-y^{2}\right)} ; \\
& \Im \bar{e}_{-2}=\Im e_{-2}=0 \\
& \Im \bar{e}_{-1}=\Im e_{-1}=0 \\
& \Im \bar{e}_{0}=\frac{2\left(1+2 y+26 y^{2}+2 y^{3}+y^{4}\right) H(0,0 ; y)}{3(1-y)(1+y)^{3}}+\frac{8 y H(-1,0,0 ; y)}{1-y^{2}} \\
& +\frac{8 y H(0,-1,0 ; y)}{1-y^{2}}-\frac{8 y H(0,0,-1 ; y)}{1-y^{2}}+\frac{2 y H(0,0,0 ; y)}{1-y^{2}} \\
& +\frac{2 H(0 ; y)}{9(1-y)^{4}(1+y)^{2}}\left[5-22 y+59 y^{2}-116 y^{3}+59 y^{4}-22 y^{5}+5 y^{6}\right. \\
& \left.+9 y \zeta_{2}-18 y^{2} \zeta_{2}+18 y^{4} \zeta_{2}-9 y^{5} \zeta_{2}\right] \\
& +\frac{4}{27(1-y)^{3}(1+y)}\left[14-40 y+28 y^{2}-40 y^{3}+14 y^{4}+81 y \zeta_{3}\right. \\
& \left.-162 y^{2} \zeta_{3}+81 y^{3} \zeta_{3}\right] \text {. }
\end{aligned}
$$

The light quark loop insertions in Fig. 2 (h) were studied before by [18,19], using a small quark mass for regularizing the double- and single logarithmic mass divergences of these contributions. As we use dimensional regularization setting the light quark masses to zero, only the coefficient of the leading singularity of the $C_{F} T_{R} N_{f}$ term in the scalar and pseudoscalar form factors, in Eqs. (71-73), (83-85) and Eqs. (95-97), (107-109) respectively, can be compared, and we agree with $[18,19]$.

The scalar and pseudoscalar $C_{F} T_{R}$ coefficients, respectively in Eqs. (74-76), (86-88) and Eqs. (98-100), (110-112), are obtained by adding the contributions coming from the diagrams in Fig. 2 (f) and (g). Our results for the latter, with the heavy quark loop insertion, agree with the ones in [19], if one takes into account that the results in [19] are listed for an on-shell renormalization of the coupling constant, while we use $\overline{\mathrm{MS}}$-renormalization for the coupling constant.

\section{Threshold Expansions}

In this section we provide the expansions of our results in the threshold limit $s \sim 4 m^{2}(y \rightarrow 1$ in the transformed variable). We define

$$
\beta=\sqrt{1-\frac{4 m^{2}}{s}}
$$


as the small expansion parameter and give the results up to the zeroth order in $\beta$.

\subsection{One-loop Scalar Case}

$$
\begin{gathered}
\Re a_{-1}=0 ; \\
\Re a_{0}=-1+\frac{3 \zeta_{2}}{\beta} ; \\
\Re a_{1}=2-\frac{6 \zeta_{2}(-1+\ln (2)+\ln (\beta))}{\beta} ; \\
\Im a_{-1}=-\frac{1}{2 \beta} ; \\
\Im a_{0}=\frac{-1+\ln (2)+\ln (\beta)}{\beta} ; \\
\Im a_{1}=\frac{1}{\beta}\left[-2+2 \ln (2)-\ln ^{2}(2)+\zeta_{2}+2 \ln (\beta)\right. \\
\left.\quad-2 \ln (2) \ln (\beta)-\ln ^{2}(\beta)\right] ;
\end{gathered}
$$

\subsection{One-loop Pseudoscalar Case}

$$
\begin{gathered}
\Re \bar{a}_{-1}=\Re a_{-1} ; \\
\Re \bar{a}_{0}=-3+\frac{3 \zeta_{2}}{\beta} ; \\
\Re \bar{a}_{1}=2-\frac{6(\ln (2)+\ln (\beta)) \zeta_{2}}{\beta} ; \\
\Im \bar{a}_{-1}=\Im a_{-1} ; \\
\Im \bar{a}_{0}=\frac{\ln (2)+\ln (\beta)}{\beta} ; \\
\Im \bar{a}_{1}=\frac{-\ln ^{2}(2)-2 \ln (2) \ln (\beta)-\ln ^{2}(\beta)+\zeta_{2}}{\beta} ;
\end{gathered}
$$




\subsection{Two-loop Scalar Case}

$$
\begin{aligned}
& \Re b_{-2}=-\frac{3 \zeta_{2}}{4 \beta^{2}}-\frac{3 \zeta_{2}}{2} ; \\
& \Re b_{-1}=\frac{3 \zeta_{2}(-1+\ln (2)+\ln (\beta))}{\beta^{2}}+\frac{3 \zeta_{2}(1+4 \ln (2)+4 \ln (\beta))}{2} ; \\
& \Re b_{0}=+\frac{3 \zeta_{2}}{2 \beta^{2}}\left[-4+8 \ln (2)-4 \ln ^{2}(2)+3 \zeta_{2}+8 \ln (\beta)\right. \\
& \left.-8 \ln (2) \ln (\beta)-4 \ln ^{2}(\beta)\right] \\
& -\frac{3 \zeta_{2}}{\beta} \\
& +\frac{1}{4}\left[5+98 \zeta_{2}-40 \ln (2) \zeta_{2}-48 \ln ^{2}(2) \zeta_{2}+36 \zeta_{2}{ }^{2}\right. \\
& -44 \zeta_{3}-88 \zeta_{2} \ln (\beta)-96 \ln (2) \zeta_{2} \ln (\beta) \\
& \left.-48 \zeta_{2} \ln ^{2}(\beta)\right] \\
& \Re c_{-2}=0 \text {; } \\
& \Re c_{-1}=0 \text {; } \\
& \Re c_{0}=-\frac{\zeta_{2}(-97+66 \ln (2)+66 \ln (\beta))}{6 \beta} \\
& +\frac{49}{36}-\frac{(-19+32 \ln (2)) \zeta_{2}}{2}-5 \zeta_{3}-4 \zeta_{2} \ln (\beta) ; \\
& \Re d_{-2}=0 \text {; } \\
& \Re d_{-1}=0 \text {; } \\
& \Re d_{0}=\frac{2 \zeta_{2}(-11+6 \ln (2)+6 \ln (\beta))}{3 \beta}-\frac{5}{9} ; \\
& \Re e_{-2}=0 \text {; } \\
& \Re e_{-1}=0 \text {; } \\
& \Re e_{0}=\frac{145}{9}-\frac{53 \zeta_{2}}{3}+4 \ln (2)\left(-1+4 \zeta_{2}\right) ;
\end{aligned}
$$




$$
\begin{aligned}
& \Im b_{-2}=0 ; \\
& \Im b_{-1}=-\frac{3 \zeta_{2}}{2 \beta^{2}}+\frac{1}{2 \beta}-3 \zeta_{2} \\
& \Im b_{0}=+\frac{6 \zeta_{2}(-1+\ln (2)+\ln (\beta))}{\beta^{2}}-\frac{\ln (2)+\ln (\beta)}{\beta} \\
& +\zeta_{2}(11+12 \ln (2)+12 \ln (\beta)) ; \\
& \Im c_{-2}=\frac{11}{24 \beta} \\
& \Im c_{-1}=-\frac{31}{72 \beta} \\
& \Im c_{0}=+\frac{1}{54 \beta}\left[-239+291 \ln (2)-99 \ln ^{2}(2)+291 \ln (\beta)\right. \\
& \left.-198 \ln (2) \ln (\beta)-99 \ln ^{2}(\beta)\right] \\
& +2 \zeta_{2} \\
& \Im d_{-2}=-\frac{1}{6 \beta} \\
& \Im d_{-1}=\frac{5}{18 \beta} \\
& \Im d_{0}=\frac{2}{27 \beta}\left[31-33 \ln (2)+9 \ln ^{2}(2)-33 \ln (\beta)\right. \\
& \left.+18 \ln (2) \ln (\beta)+9 \ln ^{2}(\beta)\right] ; \\
& \Im e_{-2}=0 \\
& \Im e_{-1}=0 \\
& \Im e_{0}=2
\end{aligned}
$$

\subsection{Two-loop Pseudoscalar Case}

$$
\begin{aligned}
\Re \bar{b}_{-2} & =\Re b_{-2} ; \\
\Re \bar{b}_{-1} & =\frac{3(\ln (2)+\ln (\beta)) \zeta_{2}}{\beta^{2}}+\frac{3(1+4 \ln (2)+4 \ln (\beta)) \zeta_{2}}{2} ;
\end{aligned}
$$




$$
\begin{aligned}
\Re \bar{b}_{0}= & \frac{-9 \zeta_{2}}{\beta}+\frac{3 \zeta_{2}\left(-4 \ln ^{2}(2)-8 \ln (2) \ln (\beta)-4 \ln ^{2}(\beta)+3 \zeta_{2}\right)}{2 \beta^{2}} \\
& +\frac{1}{4}\left[29-26 \zeta_{2}-24 \ln (2) \zeta_{2}-48 \ln ^{2}(2) \zeta_{2}-120 \ln (\beta) \zeta_{2}\right. \\
& \left.-96 \ln (2) \ln (\beta) \zeta_{2}-48 \ln ^{2}(\beta) \zeta_{2}+36 \zeta_{2}^{2}-72 \zeta_{3}\right]
\end{aligned}
$$

$$
\begin{aligned}
\Re \bar{c}_{-2}= & 0 ; \\
\Re \bar{c}_{-1}= & 0 ; \\
\Re \bar{c}_{0}= & -\frac{17}{12}-\frac{(-47+72 \ln (2)) \zeta_{2}}{2}-12 \ln (\beta) \zeta_{2} \\
& -\frac{(-31+66 \ln (2)+66 \ln (\beta)) \zeta_{2}}{6 \beta}-12 \zeta_{3} ;
\end{aligned}
$$

$\Re \bar{d}_{-2}=0$;

$\Re \bar{d}_{-1}=0 ;$

$$
\begin{aligned}
& \Re \bar{d}_{0}=\frac{1}{3}+\frac{2(-5+6 \ln (2)+6 \ln (\beta)) \zeta_{2}}{3 \beta} ; \\
& \Re \bar{e}_{-2}=0 ;
\end{aligned}
$$$$
\Re \bar{e}_{-1}=0 \text {; }
$$

$$
\Re \bar{e}_{0}=\frac{43}{3}-3 \zeta_{2}+12 \ln (2) \zeta_{2}-\frac{21 \zeta_{3}}{2} ;
$$

$$
\begin{aligned}
\Im \bar{b}_{-2}= & 0 ; \\
\Im \bar{b}_{-1}= & \frac{3}{2 \beta}-3 \zeta_{2}-\frac{3 \zeta_{2}}{2 \beta^{2}} ; \\
\Im \bar{b}_{0}= & \frac{-1-3 \ln (2)-3 \ln (\beta)}{\beta}+\frac{6(\ln (2)+\ln (\beta)) \zeta_{2}}{\beta^{2}} \\
& +3(5+4 \ln (2)+4 \ln (\beta)) \zeta_{2} ;
\end{aligned}
$$

$$
\Im \bar{c}_{-2}=\Im c_{-2}
$$




$$
\begin{aligned}
& \Im \bar{c}_{-1}= \Im c_{-1} ; \\
& \Im \bar{c}_{0}= \frac{1}{54 \beta}\left[-47+93 \ln (2)-99 \ln ^{2}(2)+93 \ln (\beta)\right. \\
&\left.-198 \ln (2) \ln (\beta)-99 \ln ^{2}(\beta)\right]+6 \zeta_{2} ; \\
& \Im \bar{d}_{-2}= \Im d_{-2} ; \\
& \Im \bar{d}_{-1}= \Im d_{-1} ; \\
& \Im \bar{d}_{0}= \frac{2}{27 \beta}\left[7-15 \ln (2)+9 \ln ^{2}(2)-15 \ln (\beta)\right. \\
&\left.+18 \ln (2) \ln (\beta)+9 \ln ^{2}(\beta)\right] ; \\
& \Im \bar{e}_{-2}=0 ; \\
& \Im \bar{e}_{-1}=0 ; \\
& \Im \bar{e}_{0}=3 \zeta_{2} .
\end{aligned}
$$

\section{Asymptotic Expansions}

In this section we provide the expansions of the form factors in the limit $s \gg m^{2}(y \rightarrow 0$ in the transformed variable). We define $r=s / m^{2}$ and $L=\ln (r)$ and keep terms up to the second order in $1 / r$.

\subsection{One-loop Scalar Case}

$$
\begin{aligned}
\Re a_{-1}= & \frac{-3+2 L}{r^{2}}-\frac{2}{r}-1+L ; \\
\Re a_{0}= & \frac{-19+32 L-2 L^{2}+16 \zeta_{2}}{2 r^{2}} \\
& +\frac{2(1+4 L)}{r}+\frac{-2-L^{2}+8 \zeta_{2}}{2} ; \\
\Re a_{1}= & \frac{3+456 L-96 L^{2}+4 L^{3}+768 \zeta_{2}-96 L \zeta_{2}+48 \zeta_{3}}{12 r^{2}} \\
& -\frac{2\left(-3 L+2 L^{2}-16 \zeta_{2}\right)}{r}-2+L+\frac{L^{3}}{6}-4 L \zeta_{2} \\
& +2 \zeta_{3} ;
\end{aligned}
$$




$$
\begin{aligned}
\Im a_{-1}= & -\frac{2}{r^{2}}-1 ; \\
\Im a_{0}= & \frac{2(-8+L)}{r^{2}}-\frac{8}{r}+L ; \\
\Im a_{1}= & \frac{-38+16 L-L^{2}+4 \zeta_{2}}{r^{2}} \\
& +\frac{2(-3+4 L)}{r}+\frac{-2-L^{2}+4 \zeta_{2}}{2} ;
\end{aligned}
$$

\subsection{One-loop Pseudoscalar Case}

$$
\begin{aligned}
& \Re \bar{a}_{-1}= \Re a_{-1} ; \\
& \Re \bar{a}_{0}= \frac{2(1+2 L)}{r}+\frac{-2-L^{2}+8 \zeta_{2}}{2} \\
&+\frac{-3+16 L-2 L^{2}+16 \zeta_{2}}{2 r^{2}} ; \\
& \Re \bar{a}_{1}=-2+L+\frac{L^{3}}{6}-\frac{2\left(L+L^{2}-8 \zeta_{2}\right)}{r}-4 L \zeta_{2}+2 \zeta_{3} \\
&+\frac{99+72 L-48 L^{2}+4 L^{3}+384 \zeta_{2}-96 L \zeta_{2}+48 \zeta_{3}}{12 r^{2}} ; \\
& \Im \bar{a}_{-1}=\Im a_{-1} ; \\
& \Im \bar{a}_{0}=0 ; \\
& \Im \bar{a}_{1}=0 ;
\end{aligned}
$$

\subsection{Two-loop Scalar Case}

$$
\begin{aligned}
\Re b_{-2}= & \frac{5-5 L+2 L^{2}-12 \zeta_{2}}{r^{2}}+\frac{-2(-1+L)}{r} \\
& +\frac{1-2 L+L^{2}-6 \zeta_{2}}{2} ; \\
\Re b_{-1}= & \frac{17-87 L+37 L^{2}-4 L^{3}-232 \zeta_{2}+80 L \zeta_{2}}{2 r^{2}} \\
& +\frac{-6 L+9 L^{2}-56 \zeta_{2}}{r}
\end{aligned}
$$




$$
+\frac{2-2 L+L^{2}-L^{3}-8 \zeta_{2}+20 L \zeta_{2}}{2}
$$

$$
\begin{aligned}
\Re b_{0}= & \frac{1}{120 r^{2}}\left[20235+6240 L+10080 L^{2}-1760 L^{3}+150 L^{4}\right. \\
& -58680 \zeta_{2}+41760 L \zeta_{2}-7200 L^{2} \zeta_{2}-34560 \ln (2) \zeta_{2} \\
& \left.+10224 \zeta_{2}^{2}+10320 \zeta_{3}-10560 L \zeta_{3}\right] \\
& +\frac{1}{15 r}\left[-30-360 L-125 L^{3}+480 \zeta_{2}+2580 L \zeta_{2}-30 L^{2} \zeta_{2}\right. \\
& \left.-1080 \ln (2) \zeta_{2}+78 \zeta_{2}{ }^{2}+210 \zeta_{3}-240 L \zeta_{3}\right] \\
& +\frac{29}{4}-\frac{L^{3}}{6}+\frac{7 L^{4}}{24}-8 \zeta_{2}+\frac{68 \zeta_{2}{ }^{2}}{5}-\frac{3 L^{2}\left(-1+8 \zeta_{2}\right)}{2} \\
& -14 \zeta_{3}+L\left(-3+\zeta_{2}+8 \zeta_{3}\right) ;
\end{aligned}
$$

$\Re c_{-2}=-\frac{11(-3+2 L)}{12 r^{2}}+\frac{11}{6 r} \frac{-11(-1+L)}{12} ;$

$$
\begin{aligned}
\Re c_{0}= & \frac{1}{2160 r^{2}}\left[-58815+264770 L-20010 L^{2}+2580 L^{3}-270 L^{4}\right. \\
& -23520 \zeta_{2}-63360 L \zeta_{2}+15120 L^{2} \zeta_{2}+311040 \ln (2) \zeta_{2} \\
& \left.-55296 \zeta_{2}^{2}+155160 \zeta_{3}-51840 L \zeta_{3}\right] \\
& +\frac{1}{54 r}\left[628+2274 L-261 L^{2}+126 \zeta_{2}-108 L \zeta_{2}\right. \\
& \left.+1944 \ln (2) \zeta_{2}+1296 \zeta_{3}\right] \\
& +\frac{1}{540}\left[-4345+1210 L-1005 L^{2}+165 L^{3}+8760 \zeta_{2}\right. \\
& -1980 L \zeta_{2}+270 L^{2} \zeta_{2}-1701 \zeta_{2}^{2}+4410 \zeta_{3} \\
& \left.-3510 L \zeta_{3}\right] ;
\end{aligned}
$$

$\Re d_{-2}=\frac{-3+2 L}{3 r^{2}}-\frac{2}{3 r}+\frac{-1+L}{3} ;$

$\Re d_{-1}=-\frac{5(-3+2 L)}{9 r^{2}}+\frac{10}{9 r}+\frac{-5(-1+L)}{9} ;$ 


$$
\begin{aligned}
\Re d_{0}= & \frac{477-2224 L+348 L^{2}-12 L^{3}-2568 \zeta_{2}+144 L \zeta_{2}-144 \zeta_{3}}{54 r^{2}} \\
& +\frac{8\left(-5-39 L+9 L^{2}-63 \zeta_{2}\right)}{27 r} \\
& +\frac{49-28 L+15 L^{2}-3 L^{3}-84 \zeta_{2}+36 L \zeta_{2}-36 \zeta_{3}}{27} ;
\end{aligned}
$$

$$
\Re e_{-2}=0 \text {; }
$$

$\Re e_{-1}=0 ;$

$$
\begin{aligned}
\Re e_{0}= & \frac{1}{270 r^{2}}\left[15360+9380 L-3930 L^{2}+840 L^{3}-45 L^{4}\right. \\
& +33480 \zeta_{2}-19800 L \zeta_{2}+540 L^{2} \zeta_{2}+3132 \zeta_{2}{ }^{2} \\
& \left.-10800 \zeta_{3}+6480 L \zeta_{3}\right] \\
& +\frac{1}{540 r}\left[4640+4560 L-720 L^{2}+45 L^{4}+2880 \zeta_{2}\right. \\
& \left.-540 L^{2} \zeta_{2}-3132 \zeta_{2}{ }^{2}-6480 L \zeta_{3}\right] \\
& +\frac{407-56 L+15 L^{2}-3 L^{3}-252 \zeta_{2}+36 L \zeta_{2}}{27}
\end{aligned}
$$

$$
\begin{aligned}
\Im b_{-2}= & \frac{5-4 L}{r^{2}}+\frac{2}{r}+1-L ; \\
\Im b_{-1}= & \frac{87-74 L+12 L^{2}-32 \zeta_{2}}{2 r^{2}}-\frac{6(-1+3 L)}{r} \\
& +1-L+\frac{3 L^{2}}{2}-4 \zeta_{2} ; \\
\Im b_{0}= & \frac{-52-168 L+44 L^{2}-5 L^{3}-172 \zeta_{2}+60 L \zeta_{2}+88 \zeta_{3}}{r^{2}} \\
& +\frac{24+25 L^{2}-72 \zeta_{2}+4 L \zeta_{2}+16 \zeta_{3}}{r} \\
& +3+\frac{L^{2}}{2}-\frac{7 L^{3}}{6}+\zeta_{2}+L\left(-3+10 \zeta_{2}\right)-8 \zeta_{3} ; \\
\Im c_{-2}= & \frac{11}{6 r^{2}}+\frac{11}{12} ; \\
\Im c_{-1}= & \frac{-47+18 L-9 L^{2}+18 \zeta_{2}}{9 r^{2}}-\frac{67}{36}+\frac{\zeta_{2}}{2} ; \\
\Im c_{0}= & \frac{1}{216 r^{2}}\left[-26477+4002 L-774 L^{2}+108 L^{3}+3240 \zeta_{2}\right.
\end{aligned}
$$




$$
\begin{aligned}
- & \left.1728 L \zeta_{2}+5184 \zeta_{3}\right] \\
+ & \frac{-379+87 L+18 \zeta_{2}}{9 r} \\
+ & \frac{-242+402 L-99 L^{2}-108 L \zeta_{2}+702 \zeta_{3}}{108} ; \\
\Im d_{-2}= & -\frac{2}{3 r^{2}}-\frac{1}{3} ; \\
\Im d_{-1}= & \frac{10}{9 r^{2}}+\frac{5}{9} ; \\
\Im d_{0}= & +\frac{2\left(556-174 L+9 L^{2}\right)}{27 r^{2}}-\frac{8(-13+6 L)}{9 r} \\
& +\frac{28-30 L+9 L^{2}}{27} ;
\end{aligned}
$$

\subsection{Two-loop Pseudoscalar Case}

$$
\begin{aligned}
\Re \bar{b}_{-2}= & \frac{-2(-1+L)}{r}+\frac{5-5 L+2 L^{2}-12 \zeta_{2}}{r^{2}}+\frac{1-2 L+L^{2}-6 \zeta_{2}}{2} ; \\
\Re \bar{b}_{-1}= & \frac{-2 L+5 L^{2}-32 \zeta_{2}}{r} \\
& +\frac{1-39 L+21 L^{2}-4 L^{3}-136 \zeta_{2}+80 L \zeta_{2}}{2 r^{2}} \\
& +\frac{2-2 L+L^{2}-L^{3}-8 \zeta_{2}+20 L \zeta_{2}}{2} ; \\
\Re \bar{b}_{0}= & \frac{29}{4}-\frac{L^{3}}{6}+\frac{7 L^{4}}{24}-8 \zeta_{2}+\frac{68 \zeta_{2}^{2}}{5}-\frac{3 L^{2}\left(-1+8 \zeta_{2}\right)}{2} \\
& -14 \zeta_{3}+L\left(-3+\zeta_{2}+8 \zeta_{3}\right) \\
& +\frac{1}{15 r}\left[-30-180 L-30 L^{2}-65 L^{3}+240 \zeta_{2}+1320 L \zeta_{2}\right.
\end{aligned}
$$




$$
\begin{aligned}
& \left.-30 L^{2} \zeta_{2}-360 \ln (2) \zeta_{2}+78 \zeta_{2}^{2}-90 \zeta_{3}-240 L \zeta_{3}\right] \\
& +\frac{1}{24 r^{2}}\left[1935+480 L+96 L^{2}-224 L^{3}+38 L^{4}-24 \zeta_{2}\right. \\
& +4512 L \zeta_{2}-1536 L^{2} \zeta_{2}-2304 \ln (2) \zeta_{2}+1872 \zeta_{2}^{2} \\
& \left.-624 \zeta_{3}-192 L \zeta_{3}\right]
\end{aligned}
$$

$$
\Re \bar{c}_{-2}=\frac{-11(-1+L)}{12}-\frac{11(-3+2 L)}{12 r^{2}}+\frac{11}{6 r} ;
$$

$$
\begin{aligned}
\Re \bar{c}_{-1}= & \frac{-67+18 \zeta_{2}}{18 r} \\
& +\frac{-165+188 L-36 L^{2}+12 L^{3}+234 \zeta_{2}-216 L \zeta_{2}-72 \zeta_{3}}{36 r^{2}} \\
& +\frac{-49+67 L+18 \zeta_{2}-18 L \zeta_{2}-18 \zeta_{3}}{36} ;
\end{aligned}
$$

$$
\begin{aligned}
\Re \bar{c}_{0}= & \frac{628+1182 L-171 L^{2}+594 \zeta_{2}-108 L \zeta_{2}+648 \ln (2) \zeta_{2}+756 \zeta_{3}}{54 r} \\
& +\frac{1}{2160 r^{2}}\left[-29055+114050 L-17130 L^{2}+5460 L^{3}-630 L^{4}\right. \\
& +48480 \zeta_{2}-97920 L \zeta_{2}+32400 L^{2} \zeta_{2}+103680 \ln (2) \zeta_{2} \\
& \left.-81216 \zeta_{2}^{2}+86040 \zeta_{3}-34560 L \zeta_{3}\right] \\
& +\frac{1}{540}\left[-4345+1210 L-1005 L^{2}+165 L^{3}+8760 \zeta_{2}-1980 L \zeta_{2}\right. \\
& \left.+270 L^{2} \zeta_{2}-1701 \zeta_{2}{ }^{2}+4410 \zeta_{3}-3510 L \zeta_{3}\right] ;
\end{aligned}
$$

$\Re \bar{d}_{-2}=\frac{-1+L}{3}+\frac{-3+2 L}{3 r^{2}}-\frac{2}{3 r} ;$

$$
\Re \bar{d}_{-1}=\frac{-5(-1+L)}{9}-\frac{5(-3+2 L)}{9 r^{2}}+\frac{10}{9 r} \text {; }
$$

$$
\begin{aligned}
\Re \bar{d}_{0}= & \frac{4\left(-10-30 L+9 L^{2}-54 \zeta_{2}\right)}{27 r} \\
& +\frac{-3-880 L+204 L^{2}-12 L^{3}-1416 \zeta_{2}+144 L \zeta_{2}-144 \zeta_{3}}{54 r^{2}} \\
& +\frac{49-28 L+15 L^{2}-3 L^{3}-84 \zeta_{2}+36 L \zeta_{2}-36 \zeta_{3}}{27} ;
\end{aligned}
$$

$\Re \bar{e}_{-2}=0 ;$

$\Re \bar{e}_{-1}=0$; 


$$
\begin{aligned}
\Re \bar{e}_{0}= & \frac{407-56 L+15 L^{2}-3 L^{3}-252 \zeta_{2}+36 L \zeta_{2}}{27} \\
& +\frac{1}{540 r}\left[1760-240 L-360 L^{2}+45 L^{4}+720 \zeta_{2}-540 L^{2} \zeta_{2}\right. \\
& \left.-3132 \zeta_{2}{ }^{2}-6480 L \zeta_{3}\right] \\
& +\frac{1}{270 r^{2}}\left[7920+5300 L+750 L^{2}-1320 L^{3}+45 L^{4}-3240 \zeta_{2}\right. \\
& \left.+14760 L \zeta_{2}-540 L^{2} \zeta_{2}-3132 \zeta_{2}{ }^{2}+6480 \zeta_{3}-6480 L \zeta_{3}\right]
\end{aligned}
$$

$$
\begin{aligned}
\Im \bar{b}_{-2}= & 1-L+\frac{5-4 L}{r^{2}}+\frac{2}{r} \\
\Im \bar{b}_{-1}= & 1-L+\frac{3 L^{2}}{2}-\frac{2(-1+5 L)}{r}+\frac{39-42 L+12 L^{2}-32 \zeta_{2}}{2 r^{2}}-4 \zeta_{2} \\
\Im \bar{b}_{0}= & 3+\frac{L^{2}}{2}-\frac{7 L^{3}}{6}+\zeta_{2}+L\left(-3+10 \zeta_{2}\right)-8 \zeta_{3} \\
& +\frac{12+4 L+13 L^{2}-36 \zeta_{2}+4 L \zeta_{2}+16 \zeta_{3}}{r} \\
& +\frac{-60-24 L+84 L^{2}-19 L^{3}-228 \zeta_{2}+156 L \zeta_{2}+24 \zeta_{3}}{3 r^{2}}
\end{aligned}
$$

$$
\begin{aligned}
\Im \bar{d}_{-2} & =-\frac{1}{3}-\frac{2}{3 r^{2}} \\
\Im \bar{d}_{-1} & =\frac{5}{9}+\frac{10}{9 r^{2}} \\
\Im \bar{d}_{0} & =\frac{28-30 L+9 L^{2}}{27}+\frac{2\left(220-102 L+9 L^{2}\right)}{27 r^{2}}-\frac{8(-5+3 L)}{9 r} ;
\end{aligned}
$$




$$
\begin{aligned}
\Im \bar{e}_{-2}= & 0 ; \\
\Im \bar{e}_{-1}= & 0 ; \\
\Im \bar{e}_{0}= & \frac{56-30 L+9 L^{2}}{27}+\frac{4+12 L-3 L^{3}-18 L \zeta_{2}+108 \zeta_{3}}{9 r} \\
& -\frac{2\left(265+75 L-198 L^{2}+9 L^{3}-54 \zeta_{2}+54 L \zeta_{2}-324 \zeta_{3}\right)}{27 r^{2}} .
\end{aligned}
$$

\section{Summary and Outlook}

If Higgs boson will be discovered, then the next important task will be the experimental determination of its couplings to the other known fundamental particles, in particular to heavy quarks $Q=c, b, t$. Therefore precise predictions of Higgs boson decays into heavy quark-antiquark pairs are mandatory, not only for the partial decay rates, but also for differential distributions.

To put forward this aim we have computed the two-loop QCD corrections to the decay amplitude of a neutral Higgs boson, with scalar and pseudoscalar couplings to fermions, into a heavy quark-antiquark pair, $h \rightarrow Q \bar{Q}$. This amplitude is determined by two form factors, which we calculated with the algebraic and analytic techniques employed in $[35,36,39,44,45]$, for arbitrary quark mass $m$ and squared four-momentum $q^{2}$ of the spin-zero particle. These scalar and pseudoscalar vertex functions have also a number of other applications, including resonant Higgs boson production in high energetic muon collisions, $\mu^{+} \mu^{-} \rightarrow Q \bar{Q} X$. Applications to hadron physics include matrix elements of scalar and pseudoscalar quark currents, serving as interpolating fields for heavy mesons, between heavy (anti)quark states. Moreover, from the results presented here one obtains as a special case also the second order QED corrections for the amplitudes $h \rightarrow f \bar{f}, f$ being a quark or a lepton.

In addition, we expanded the scalar and pseudoscalar form factors near the $Q \bar{Q}$ threshold and in the regime $m^{2} / q^{2} \ll 1$. The former expansions may be applied, for instance, to the decay of heavy nonstandard Higgs bosons into $t \bar{t}$, the latter ones apply to, e.g., $h \rightarrow b \bar{b}, c \bar{c}$.

The renormalized form factors were expressed in terms of the $\overline{\mathrm{MS}}$ coupling $\alpha_{S}$ for $\left(N_{f}+\right.$ 1) quark flavors and the on-shell mass $m$ of the heavy quark. They may be expressed in straightforward fashion as functions of the running $\overline{\mathrm{MS}}$ mass $\bar{m}(\mu)$, too. It is expected that the use of this mass parameter leads to a faster decrease of the coefficients of the perturbation series [4]. However, this is no general rule (see, e.g., [14]); the size of the coefficients of the series when using $m$ or $\bar{m}$ must be studied for each observable separately.

The renormalized form factors contain soft and collinear divergences, which in our IRregularization scheme appear as poles in $\epsilon$. These divergences have to be canceled against the divergences arising from the real radiation of gluons and massless quark-antiquark pairs, which in this paper were not taken into account. The IR singularity structure of QCD amplitudes involving massive quarks is so far not understood beyond the 1-loop level. The presently known two-loop heavy-quark vertex functions may provide insight into the generic singularity structure of the two-loop QCD amplitudes with massive quarks. As to the two-loop scalar and pseudoscalar form factors given above we notice that the infrared-divergent contributions proportional to the colour factor $C_{F}^{2}$ show Yenni-Frautschi-Suura exponentiation [50], which 
is characteristic of Abelian gauge theories. This is completely analogous to the case of the two-loop QCD vector and axial vector vertex functions [36]. However the IR-divergent terms proportional to the other colour factors cannot be explained by naive Abelian exponentiation. It remains an open problem whether an infrared factorization formula (as derived for massless QCD in $[51,52])$ exists for the generation of these singularities beyond the one-loop level.

As emphasized, the results of this paper are one building block towards a differential description of Higgs boson decays, $h \rightarrow Q \bar{Q} X$, to second order QCD.

\section{Acknowledgment}

This work was partially supported by Deutsche Forschungsgemeinschaft (DFG), SFB/TR9, by DFG-Graduiertenkolleg RWTH Aachen, by the Swiss National Science Foundation (SNF) under contract 200021-101874, by the National Science Foundation under Grant No. PHY99-07949, and by the USA DoE under the grant DE-FG03-91ER40662, Task J.

\section{References}

[1] A. Djouadi, arXiv:hep-ph/0503172.

[2] A. Djouadi, arXiv:hep-ph/0503173.

[3] G. Weiglein et al. [LHC/LC Study Group], arXiv:hep-ph/0410364.

[4] E. Braaten and J. P. Leveille, Phys. Rev. D 22 (1980) 715.

[5] N. Sakai, Phys. Rev. D 22 (1980) 2220.

[6] T. Inami and T. Kubota, Nucl. Phys. B 179 (1981) 171.

[7] M. Drees and K. I. Hikasa, Phys. Lett. B 240 (1990) 455 [Erratum-ibid. B 262 (1991) 497].

[8] S. G. Gorishnii, A. L. Kataev and S. A. Larin, Sov. J. Nucl. Phys. 40 (1984) 329 [Yad. Fiz. 40 (1984) 517].

[9] S. G. Gorishnii, A. L. Kataev, S. A. Larin and L. R. Surguladze, Phys. Rev. D 43 (1991) 1633.

[10] L. R. Surguladze, Phys. Lett. B 338 (1994) 229 arXiv:hep-ph/9406294.

[11] L. R. Surguladze, Phys. Lett. B 341 (1994) 60 arXiv:hep-ph/9405325.

[12] S. A. Larin, T. van Ritbergen and J. A. M. Vermaseren, Phys. Lett. B 362 (1995) 134 arXiv:hep-ph/9506465.

[13] K. G. Chetyrkin and A. Kwiatkowski, Nucl. Phys. B 461 (1996) 3 arXiv:hep-ph/9505358.

[14] R. Harlander and M. Steinhauser, Phys. Rev. D 56 (1997) 3980 arXiv:hep-ph/9704436.

[15] R. V. Harlander and W. B. Kilgore, Phys. Rev. D 68 (2003) 013001 arXiv:hep-ph/0304035.

[16] K. G. Chetyrkin and M. Steinhauser, Phys. Lett. B 408 (1997) 320 arXiv: hep-ph/9706462.

[17] K. G. Chetyrkin, B. A. Kniehl, M. Steinhauser and W. A. Bardeen, Nucl. Phys. B 535, 3 (1998) arXiv:hep-ph/9807241.

[18] K. Melnikov, Phys. Rev. D 53 (1996) 5020 arXiv:hep-ph/9511310.

[19] A. H. Hoang and T. Teubner, Nucl. Phys. B 519, 285 (1998) arXiv:hep-ph/9707496. 
[20] J. Fleischer and F. Jegerlehner, Phys. Rev. D 23 (1981) 2001.

[21] D. Y. Bardin, B. M. Vilensky and P. K. Khristova, Sov. J. Nucl. Phys. 53 (1991) 152 [Yad. Fiz. 53 (1991) 240].

[22] B. A. Kniehl, Nucl. Phys. B 376 (1992) 3.

[23] A. Dabelstein and W. Hollik, Z. Phys. C 53 (1992) 507.

[24] A. Djouadi, P. Gambino and B. A. Kniehl, Nucl. Phys. B 523 (1998) 17 arXiv:hep-ph/9712330.

[25] L. Durand, B. A. Kniehl and K. Riesselmann, Phys. Rev. D 51 (1995) 5007 arXiv:hep-ph/9412311.

[26] A. Ghinculov, Phys. Lett. B 337 (1994) 137 [Erratum-ibid. B 346 (1995) 426] arXiv:hep-ph/9405394.

[27] A. Dabelstein, Nucl. Phys. B 456 (1995) 25 arXiv:hep-ph/9503443.

[28] H. Eberl, K. Hidaka, S. Kraml, W. Majerotto and Y. Yamada, Phys. Rev. D 62 (2000) 055006 arXiv:hep-ph/9912463.

[29] S. Heinemeyer, W. Hollik and G. Weiglein, Eur. Phys. J. C 16 (2000) 139 arXiv:hep-ph/0003022.

[30] J. Guasch, P. Häfliger and M. Spira, Phys. Rev. D 68 (2003) 115001 arXiv:hep-ph/0305101.

[31] W. Bernreuther and A. Brandenburg, Phys. Lett. B 314 (1993) 104.

[32] W. Bernreuther, A. Brandenburg and M. Flesch, Phys. Rev. D 56 (1997) 90 arXiv:hep-ph/9701347.

[33] W. Bernreuther, A. Brandenburg and M. Flesch, arXiv:hep-ph/9812387.

[34] A. Gehrmann-De Ridder, T. Gehrmann and E. W. N. Glover, arXiv:hep-ph/0505111.

[35] W. Bernreuther, R. Bonciani, T. Gehrmann, R. Heinesch, T. Leineweber and E. Remiddi, Nucl. Phys. B 723 (2005) 91 arXiv:hep-ph/0504190.

[36] W. Bernreuther, R. Bonciani, T. Gehrmann, R. Heinesch, T. Leineweber, P. Mastrolia and E. Remiddi, Nucl. Phys. B 706, 245 (2005) arXiv:hep-ph/0406046.

W. Bernreuther, R. Bonciani, T. Gehrmann, R. Heinesch, T. Leineweber, P. Mastrolia and E. Remiddi, Nucl. Phys. B 712 (2005) 229 arXiv:hep-ph/0412259.

[37] D. J. Broadhurst, N. Gray and K. Schilcher, Z. Phys. C52 (1991) 111.

[38] K. Melnikov and T. van Ritbergen, Nucl. Phys. B591 (2000) 515 [arXiv:hep-ph/0005131].

[39] R. Bonciani, P. Mastrolia and E. Remiddi, Nucl. Phys. B676 (2004) 399 [arXiv:hep-ph/0307295] .

[40] J.A.M. Vermaseren, Symbolic Manipulation with FORM, Version 2, CAN, Amsterdam, 1991; New features of FORM arXiv:math-ph/0010025.

[41] S. Laporta and E. Remiddi, Phys. Lett. B379 (1996) 283 [arXiv:hep-ph/9602417] .

S. Laporta, Int. J. Mod. Phys. A 15 (2000) 5087 [arXiv:hep-ph/0102033] .

C. Anastasiou and A. Lazopoulos, JHEP 0407 (2004) 046 arXiv:hep-ph/0404258.

[42] F.V. Tkachov, Phys. Lett. B100 (1981) 65.

K.G. Chetyrkin and F.V. Tkachov, Nucl. Phys. B192 (1981) 159.

[43] T. Gehrmann and E. Remiddi, Nucl. Phys. B580 (2000) 485 arXiv:hep-ph/9912329.

[44] R. Bonciani, P. Mastrolia and E. Remiddi, Nucl. Phys. B661 (2003) 289 [arXiv:hep-ph/0301170].

[45] R. Bonciani, P. Mastrolia and E. Remiddi, Nucl. Phys. B690 (2004) 138 
[arXiv:hep-ph/0311145] .

[46] G. 't Hooft and M. Veltman, Nucl. Phys. B44 (1972) 189.

C. G. Bollini and J. J. Giambiagi, Phys. Lett. B40 (1972) 566; Nuovo Cim. 12B (1972) 20.

J. Ashmore, Lett. Nuovo Cimento 4 (1972) 289.

G. M. Cicuta and E. Montaldi, Lett. Nuovo Cimento 4 (1972) 289.

R. Gastmans and R. Meuldermans, Nucl. Phys. B63 (1973) 277.

[47] A. V. Kotikov, Phys. Lett. B254 (1991) 158.

A. V. Kotikov, Phys. Lett. B259 (1991) 314.

A. V. Kotikov, Phys. Lett. B267 (1991) 123.

E. Remiddi, Nuovo Cim. 110A (1997) 1435 arXiv:hep-th/9711188.

M. Caffo, H. Czyż, S. Laporta and E. Remiddi, Acta Phys. Polon. B29 (1998) 2627 arXiv:hep-ph/9807119.

M. Caffo, H. Czyż, S. Laporta and E. Remiddi, Nuovo Cim. A111 (1998) 365 arXiv:hep-ph/9805118.

[48] A.B.Goncharov, Math. Res. Lett. 5 (1998), 497-516.

D. J. Broadhurst, Eur. Phys. J. C 8 (1999) 311 arXiv:hep-th/9803091.

E. Remiddi and J. A. M. Vermaseren, Int. J. Mod. Phys. A15 (2000) 725 arXiv:hep-ph/9905237.

[49] T. Gehrmann and E. Remiddi, Comput. Phys. Commun. 141 (2001) 296 arXiv:hep-ph/0107173.

D. Maître, arXiv:hep-ph/0507152.

[50] D.R. Yennie, S.C. Frautschi and H. Suura, Annals Phys. 13 (1961) 379.

[51] S. Catani, Phys. Lett. B 427 (1998) 161 arXiv:hep-ph/9802439.

[52] G. Sterman and M. E. Tejeda-Yeomans, Phys. Lett. B $552 \quad$ (2003) 48 arXiv:hep-ph/0210130. 